\title{
The lightest scalar glueball
}

\author{
V.V. Anisovich \\ TO THE MEMORY OF YURI DMITRIEVICH PROKOSHKIN
}

\begin{abstract}
Recently performed investigations of meson spectra allow us to determine the resonance structure for the waves $I J^{P C}=00^{++}, 10^{++}, 02^{++}, 12^{++}=I J^{P}=\frac{1}{2} 0^{+}$in the mass region up to 1900 $\mathrm{MeV}$, thus establishing the meson multiplets $1^{3} P_{0} q \bar{q}$ and $2^{3} P_{0} q \bar{q}$. Experimental data demonstrate that there are five scalar/isoscalar states in this mass region. Four of them are $q \bar{q}$ states, that is, members of the $1^{3} P_{0} q \bar{q}$ and $2^{3} P_{0} q \bar{q}$ nonets, while the fifth state is an extra one not accomodated by $q \bar{q}$ systematics; it has the properties of the lightest scalar glueball. Analysis of the $00^{++}$-wave performed within the framework of the dispersion relation technique allows us to reconstruct the mixing of a pure gluonium with neighbouring scalar $q \bar{q}$ states belonging to $1^{3} P_{0} q \bar{q}$ and $2^{3} P_{0} q \bar{q}$ nonets: three scalar mesons share the gluonium state between each other - those are two comparatively narrow resonances $f_{0}(1300)$ and $f_{0}(1500)$ and a broad resonance $f_{0}\left(1530_{-250}^{+90}\right)$. The broad state is a descendant of the gluonium, keeping about $40-50 \%$ of its component.
\end{abstract}

\section{Introduction: retrospective view and the current state of the problem}

A great variety of the currently observed mesons and baryons represent systems built of quarks. These are baryons, which are three-quark systems $(q q q)$, and mesons, which are quark-antiquark bound states $(q \bar{q})$. More than 20 years ago the problem arose [1] whether additional hadrons exist which are built out of another fundamental QCD particle, the gluon. An intensive search for the glueball - a particle consisting of gluons - was carried out throughout these decades.

First evaluation of the glueball masses for different $J^{P C}$ was done in the bag model [2]. According to it, the lightest glueballs are scalars and tensors, $0^{++}$and $2^{++}$; then follow pseudoscalar and pseudotensor glueballs, $0^{-+}$and $2^{-+}$.

Recently considerable progress has been achieved in Lattice QCD calculations. The UKQCD collaboration [3] obtained the following mass values for the lightest gluodynamical glueballs (i.e. the glueball without quark degrees of freedom taken into account):

$$
m_{G}\left(0^{++}\right)=1549 \pm 53 \mathrm{MeV}, \quad m_{G}\left(2^{++}\right)=2310 \pm 110 \mathrm{MeV}, \quad m_{G}\left(0^{-+}\right)=2332 \pm 264 \mathrm{MeV} .
$$

Systematic errors are not included into the values given in Eq. (1.1); they are of the order of 100 $\mathrm{MeV}$.

The IBM group obtained a slightly different value for the mass of the lightest scalar glueball [⿴囗十

$$
m_{G}\left(0^{++}\right)=1740 \pm 71 \mathrm{MeV},
$$

The result of Ref. [5] is as follows:

$$
m_{G}\left(0^{++}\right)=1630 \pm 60 \pm 80 \mathrm{MeV}, \quad m_{G}\left(2^{++}\right)=2400 \pm 10 \pm 120 \mathrm{MeV} .
$$


However, in Lattice calculations cited above the quark degrees of freedom have not been taken into account, since the existing computing facilities did not allow it. Quark degrees of freedom may shift the position of the glueball mass noticeably. The dispersion relation analysis of meson spectra [6, 7], based on a restoration of the propagator matrix for scalar/isoscalar resonances, shows that the mixing with $q \bar{q}$-states results in a mass shift of the order of $100-300 \mathrm{MeV}$. It should be stressed that, according to the $1 / N$ expansion rules [8] $\left(N=N_{f}=N_{c}\right.$, where $N_{f}$ and $N_{c}$ stand for the light flavour and colour numbers), the mixing of a glueball with $q \bar{q}$ states is not suppressed.

Experimental searches for the glueballs were particularly intensive during the last decade. There exist reactions where one could expect an enhanced production of glueballs. Central hadron production at the high-energy hadron-hadron collisions provides us with an example of such a reaction, because the particles in the central region are produced in the transition pomerons $\rightarrow$ hadrons. The Pomeron is a gluon-rich system, so one could expect to find among secondary hadrons dominant production of glueballs, while the production of $q \bar{q}$-states is expected to be small. However, the data on central hadron production, with statistics sufficient to perform a reliable partial wave analysis, are only now appearing. More accessible for experimental study appeared to be another reaction, which is governed by the transition gluons $\rightarrow$ hadrons; this is, radiative decays $J / \psi \rightarrow \gamma+$ hadrons . In these decays, hadrons are formed by gluons created in $c \bar{c}$ annihilation; therefore one may expect dominant production of glueballs in this reaction. Experimental study of hadron spectra in radiative $J / \psi$ decays has been carried out during 2 decades, and is still going on. Experimental information accumulated at the beginning of the 90 's seemed to be rather discouraging, for in radiative $J / \psi$ decays $q \bar{q}$ states have been strongly produced: meson production branching ratios presented in the PDG compilation [9] show a number of resonances produced with similar probabilities, such as $\eta, \eta^{\prime}, f_{2}(1270), f_{2}(1525)$, etc., which certainly are $q \bar{q}$-dominant systems. Such a situation presents a dilemma:

(1) The glueball does not exist; it is "an unfulfilled promise of QCD" 10.

(2) Glueball states are mixed strongly with the $q \bar{q}$ mesons, so in experiments one observes just these mixed states.

Analysis of the $00^{++}$wave [6, 7] definitely supports the second scenario.

Experimental data on the transition form factors $\gamma \gamma^{*}\left(Q^{2}\right) \rightarrow \pi^{0}, \eta, \eta^{\prime}$ 11 provide the following restrictions for probabilities to find the glueball components in $\eta$ and $\eta^{\prime}$ mesons: $W_{\eta} \leq 8 \%, W_{\eta^{\prime}} \leq 20 \%$ 12. This means that in the $q \bar{q}$ mesons observed in radiative $J / \psi$ decay one could find a glueball component at the level of $5-10 \%$. Hence, the admixture of the $q \bar{q}$ component in the glueball should be considerably more, since the glueball can mix with several $q \bar{q}$ mesons. This qualitative estimation agrees with that obtained in the framework of the $1 / N$ expansion: according to this, the glueball component in each $q \bar{q}$ meson is of the order of $1 / N_{c}$, while the $q \bar{q}$ component in the glueball is of the order of $N_{f} / N_{c}$ 13]. Of course, it should be stressed that some specific cases may differ from this general evaluation, because the mixing depends strongly on the relative spacing of mixed levels.

If the scenario (2) with strong mixing of the glueball and $q \bar{q}$ states is realized in Nature, the search for the glueball is laborious and difficult work involving the identification of mesons and their systematics. Naive expectations, such as a study of the gluon-rich reactions with the purpose of seeing direct glueball production or, conversely, a study of hadron production in $\gamma \gamma \rightarrow q \bar{q}$-induced reactions with the hope of excluding gluon-rich hadrons, cannot be expected to succeed.

The main channel of radiative $J / \psi$ decays, as deduced from experimental data, is the production of broad hadron clusters. The production of these clusters may be viewed as a direct signal of strong mixing between the glueball and $q \bar{q}$ mesons. What happens is that, through mixing, one resonance accumulates the widths of other resonances. This effect has been first observed in [14], where the low-energy part of the spectrum of the $00^{++}$wave has been analysed; this effect has been investigated in detail [6, 7]. When the two resonances mix completely with each other, one of them gets almost the whole width $\Gamma_{1}+\Gamma_{2}$, while the width of the other one tends to zero. In the case of an "ideal" mixing of three resonances, the width of one of them accumulates the widths of the other two, $\Gamma_{1}+\Gamma_{2}+\Gamma_{3}$, 
and the widths of the others tend to zero. In reality, when the scalar glueball mixes with neighbouring states, there occurs a qualitatively similar effect; that is, a glueball situated among the scalar $q \bar{q}$ states mixes with them and accumulates a considerable part of their widths. From this point of view, the appearance of a broad resonance which is the glueball descendant is an inevitable consequence of the mixing. The broad resonance must be a neighbour of comparatively narrow resonances, which are the descendant of pure $q \bar{q}$ states; the broad resonance contains a considerable glueball admixture. The analysis of the $00^{++}$wave in the mass range $1200-1800 \mathrm{MeV}$, based on the dispersion relation representation, reconstructs just this picture of the lightest scalar glueball mixing with $q \bar{q}$ members of multiplets $1^{3} P_{0}$ and $2^{3} P_{0}$. One may predict that such a scenario of mixing is common for all low-lying glueballs.

Thus, the strong mixing of $q \bar{q}$ states with a gluonium does not allow easy identification of the glueball. In this case the only reasonable strategy is to study the systematics of all resonances in terms of $q \bar{q}$ multiplets. The extra states which do not fit into $q \bar{q}$ systematics should be regarded as candidates for the glueballs or other exotic mesons. This investigation program has been declared in [13, and at the same time the first steps have been made in carrying it out: in Ref. [14] the $K$-matrix analysis has been performed for the low-energy part of the wave $I J^{P C}=00^{++}$.

Detailed analysis of meson states in the region 1000-2000 MeV was possible due to the huge sample of experimental data collected in the latest decade by Crystal Barrel and GAMS Collaborations. The Crystal Barrel Collaboration has high-precision data on the production of three neutral mesons in the reaction $p \bar{p}$ annihilation at rest,

$$
p \bar{p}(\text { at rest }) \rightarrow \pi^{0} \pi^{0} \pi^{0}, \quad \pi^{0} \pi^{0} \eta, \quad \pi^{0} \eta \eta,
$$

with the event numbers 720000 for $\left(\pi^{0} \pi^{0} \pi^{0}\right), 280000$ for $\left(\pi^{0} \pi^{0} \eta\right)$ and 185000 for $\left(\pi^{0} \eta \eta\right)$. The data on the reaction $p \bar{p}$ (at rest) $\rightarrow \pi^{0} \pi^{0} \pi^{0}$, with somewhat lower statistics were published in 1991 [15]. However, the first fits of the spectra did not provide a correct identification of scalar resonances, for certain special features of the three-particle decay were not taken into account. A critical analysis of the situation has been made in [16, 17], where it was shown that a resonance near $1500 \mathrm{MeV}$, which had earlier been identified as a tensor one, $A X_{2}(1520)$, actually is a scalar resonance. Reanalysis of the reactions (1.4) within the $T$-matrix formalism performed together with the Crystal Barrel Collaboration has fixed the existence of new scalar resonances: $f_{0}(1500)$ [18] and $a_{0}(1450)$ [19. In addition, in [16, 17, 18] a noticeable production of the resonance $f_{0}(1360)$, with a half-width equal to $130 \mathrm{MeV}$, weas identified, although at that time it was not quite clear whether this was a newly observed resonance or a fragment of the broad resonance $\epsilon(1300)$, which was widely discussed during the latest decades. Later on, after having performed the $K$-matrix analysis for larger samples of data, it became clear that in this mass region there are two resonances - a comparatively narrow one, $f_{0}(1360)$, and a rather broad one , $f_{0}\left(1530_{-250}^{+90}\right)$.

In the first stage of the investigation, the fitting to data was done in the framework of the $T$ matrix technique. The reason was obvious: the $T$-matrix representation of the amplitude is simpler for fitting to data; the advantages of the $K$-matrix approach reveal themselves only when there exists information on all possible channels of the reaction. In the mass region 1000-1500 MeV, there are the following channels in the $00^{++}$wave: $\pi \pi, K \bar{K}, \eta \eta$ and $\pi \pi \pi \pi$, while in the region above $1500 \mathrm{MeV}$ the channel $\eta \eta^{\prime}$ becomes important. It was obvious that the application of the sophisticated $K$-matrix technique for fitting to a limited number of channels (1.4) would lead to some ambiguities.

The discovery of the resonance $f_{0}(1500)$ immediately gave rise to the hypotheses of its close relation to the lightest scalar glueball, and the possibility of such a relation was stressed in [17, 18. In the following papers $13,20,21,22,23$, several schemes have been suggested for the mixing of the lightest scalar glueball with the neighbouring $q \bar{q}$-states. However, all these schemes did not take account of special features of the mixing which are due to the transition of a resonance into real mesons, although just these transitions, as was shown in a specified $K$-matrix analysis, determine the structure of the $00^{++}$-wave around $1500 \mathrm{MeV}$. 
At the next stage of the $00^{++}$wave analysis, the GAMS data on the spectra $\pi^{0} \pi^{0}, \eta \eta$ and $\eta \eta^{\prime}$ have been included; these were obtained in the reactions [24, 25, 26]:

$$
\pi^{-} p \rightarrow n \pi^{0} \pi^{0}, \quad n \eta \eta, \quad n \eta \eta^{\prime},
$$

together with the data of the CERN-Münich collaboration [27]:

$$
\pi^{-} p \rightarrow n \pi^{+} \pi^{-}
$$

and BNL [28] group:

$$
\pi \pi \rightarrow K \bar{K}
$$

Simultaneous analysis of the whole data sample (1.4)-(1.7) was carried out in Refs. [14, 29, 30], in the framework of the $K$-matrix technique; in this way the range of masses under investigation and the number of channels covered by the $K$-matrix fit of the $00^{++}$amplitude gradually increased.

The first investigation [14] was done in the region of invariant meson energies $\sqrt{s} \leq 1100 \mathrm{MeV}$ for the two channels only, namely, $\pi \pi$ and $K \bar{K}$, In this analysis an observation, which became henceforth important, was made: the transitions which are responsible for the decay of meson states are also responsible for a strong mixing of these states. Moreover, the masses of mixed states differ essentially from the primary ones. These "primary mesons" were called in [14] "bare mesons", in contrast to physical states, for which the cloud of real particles, $\pi \pi$ and $K K$, plays an important role in their formation. The masses of bare states are defined as the $K$-matrix poles. The above-mentioned accumulation of widths of the primary states by one of the resonances due to mixing, was also observed in Ref. [14].

As the next step, the $K$-matrix analysis was extended to $1550 \mathrm{MeV}$ 29], with additional channels $\eta \eta$ and $\pi \pi \pi \pi$ included. The channel $\pi \pi \pi \pi$ is rather important for the correct description of spectra from 1300 to $1600 \mathrm{MeV}$, since $\sigma(\pi \pi \rightarrow \pi \pi \pi \pi) / \sigma(\pi \pi \rightarrow \pi \pi)$ is of the order of 0.5 at $1300 \mathrm{MeV}$ and about 1.5 at $1500 \mathrm{MeV}$ 31. The use of channels $\pi \pi, K \bar{K}$ and $\eta \eta$ provides an opportunity to perform the $q \bar{q}$ classification of bare $00^{++}$states, $f_{0}^{b a r e}$, below $1600 \mathrm{MeV}$ [29]. The point is that $q \bar{q}$-meson decays go to the new $q \bar{q}$-pair via the production of intermediate gluons. According to the rules of the $1 / N$ expansion, the main contribution to the decay coupling constant comes from planar diagrams. When an isoscalar $q \bar{q}$ - meson disintegrates into two pseudoscalar mesons $P_{1} P_{2}$, namely,

$$
\pi \pi, \quad K \bar{K}, \quad \eta \eta, \quad \eta \eta^{\prime}, \quad \eta^{\prime} \eta^{\prime},
$$

the coupling constants can be determined, up to a common factor, by two factors. The first is the quark content of the $q \bar{q}$-meson

$$
q \bar{q}=n \bar{n} \cos \phi+s \bar{s} \sin \phi,
$$

where $n \bar{n}=(u \bar{u}+d \bar{d}) / \sqrt{2}$. The second is the parameter $\lambda$, which characterises the relative probability to produce nonstrange and strange quarks by gluons in soft processes:

$$
u \bar{u}: d \bar{d}: s \bar{s}=1: 1: \lambda .
$$

Experimental data provide the following values for this parameter: $\lambda \simeq 0.5$ [32] in central hadron production in hadron-hadron high-energy collisions, $\lambda=0.8 \pm 0.2$ 33] for the decay of tensor mesons and $\lambda=0.6 \pm 0.1$ [34, 35] for the ratio of yields of $\eta$ and $\eta^{\prime}$ mesons in the decays $J / \psi \rightarrow \gamma \eta / \gamma \eta^{\prime}$.

Coupling constants for the decay $q \bar{q} \rightarrow P_{1} P_{2}$ into channels (1.8), which are defined by the leading planar diagrams in the $1 / N$ expansion, may be presented as

$$
g\left(q \bar{q} \rightarrow P_{1} P_{2}\right)=C_{P_{1} P_{2}}(\phi, \lambda) g^{L},
$$

where $C_{P_{1} P_{2}}(\phi, \lambda)$ is a wholly calculable coefficient depending on the mixing angle $\phi$ and parameter $\lambda ; g^{L}$ is a common factor describing the unknown dynamics of the process. Therefore, experimental 
investigation of resonance decays into channels (1.8) allows us to reconstruct the quark content of the state (i.e. its mixing angle $\phi$ ), thus making it possible to establish the meson systematics.

However, on the basis of the decay constant analysis, it is impossible to determine unambigously whether we deal with a $q \bar{q}$-meson or with the glueball. The reason is that the glueball decay is a two-stage process, with a subsequent production of two $q \bar{q}$ pairs. After the production of the first $q \bar{q}$ pair, in the intermediate state there exists a $q \bar{q}$ system with the following $n \bar{n} / s \bar{s}$ content:

$$
n \bar{n} \cos \phi_{\text {Glueball }}+s \bar{s} \sin \phi_{\text {Glueball }}, \quad \tan \phi_{\text {Glueball }}=\sqrt{\frac{\lambda}{2}} .
$$

For $\lambda=0.45-0.80$ the mixing angle is $\phi_{\text {Glueball }}=25^{\circ}-32^{\circ}$. At the second stage, the intermediate $q \bar{q}$ state (1.12) turns into the $P_{1} P_{2}$ mesons; this means that the relations between glueball coupling constants are the same as for the decay of the $q \bar{q}$ meson with $\phi=\phi_{\text {Glueball }}$.

Analysis of the $\pi \pi, K \bar{K}$ and $\eta \eta$ spectra performed in 29 proved that in the region below 1600 $\mathrm{MeV}$ there are four scalar/isoscalar states, and only one of them is an $s \bar{s}$-dominant state. Since each of the ${ }^{3} P_{0} q \bar{q}$ multiplets contains two $I=0$ states, which refer to two flavour combinations $n \bar{n}$ and $s \bar{s}$, then, as a result of the analysis of [29], the following dilemma becomes apparent:

1. In the region $1000-1800 \mathrm{MeV}$ there are three ${ }^{3} P_{0} q \bar{q}$ nonets: the basic one, $1^{3} P_{0} q \bar{q}$, and two radial excitations, $2^{3} P_{0} q \bar{q}$ and $3^{3} P_{0} q \bar{q}$. In this case, there should exist two $s \bar{s}$-dominant scalar mesons in the region $1600-1800 \mathrm{MeV}$.

2. At $1600-1800 \mathrm{MeV}$, there is only one $s \bar{s}$-dominant state. Then, one of the three mesons from the region $1200-1600 \mathrm{MeV}$ is an extra one from the point of view of $q \bar{q}$-systematics, and it should be considered as a candidate for an exotic meson: the ratios of couplings to the channels (1.8) found in 29 provide the basis to consider it as the lightest scalar glueball.

Thus, after carrying out the analysis of Ref. [29], the immediate task was to extend the $K$-matrix analysis of the $00^{++}$wave to the region $1600-1900 \mathrm{MeV}$. Such an extension suggested the inclusion of the $\eta \eta^{\prime}$ channel into the fitting procedure; this has been done in [30], where the $K$-matrix analysis has been performed in the mass region $500-1900 \mathrm{MeV}$, with the next five channels taken into consideration: $\pi \pi, K \bar{K}, \eta \eta, \pi \pi \pi \pi, \eta \eta^{\prime}$. It was shown that in the range $1600-1900 \mathrm{MeV}$ there exists only one $f_{0}$-meson with a dominant $s \bar{s}$ component, hence the analysis 30] confirmed the case $\mathbf{2}$. In this way, it was also shown that there are two variants for fixing the scalar glueball:

Solution I. Two bare states, $f_{0}^{\text {bare }}(720 \pm 100)$ and $f_{0}^{\text {bare }}(1260 \pm 30)$, are members of the multiplet $1^{3} P_{0} q \bar{q}$, and $f_{0}^{\text {bare }}(720)$ is the $s \bar{s}$-rich state, with $\phi(720)=-69^{\circ} \pm 12^{\circ}$. The bare states $f_{0}^{\text {bare }}(1600 \pm 50)$ and $f_{0}^{\text {bare }}(1810 \pm 30)$ are members of the $2^{3} P_{0} q \bar{q}$ nonet, and $f_{0}^{\text {bare }}(1600)$ is dominantly $n \bar{n}$-state, with $\phi(1600)=-6^{\circ} \pm 15^{\circ}$. The state $f_{0}^{\text {bare }}(1235 \pm 50)$ is superfluous from the point of view of the $q \bar{q}$ classification; its coupling constants satisfy the ratios relevant to gluonium. Therefore, this state may be considered as a candidate for the lightest scalar glueball.

Solution II. The basic scalar nonet is the same as in Solution I. The members of the next nonet, $2^{3} P_{0} q \bar{q}$, are as follows: $f_{0}^{\text {bare }}(1235 \pm 50)$ and $f_{0}^{\text {bare }}(1810 \pm 30)$. Both these states contain a considerable admixture of the $s \bar{s}$ component: $\phi(1235)=42^{\circ} \pm 10^{\circ}$ and $\phi(1810)=-53^{\circ} \pm 10^{\circ}$. The state $f_{0}^{\text {bare }}(1560 \pm 30)$ is an extra one from the point of view of $q \bar{q}$ systematics and it may be regarded as a good candidate for the lightest scalar glueball.

The existence of two variants corresponds to the impossibility to answer unambiguously, on the basis of the information on the decay channels (1.8), if we deal with the glueball or $q \bar{q}$-meson with the mixing angle $\phi$ in the region $25^{\circ}-32^{\circ}$, as was stressed above.

Both $K$-matrix solutions, I and II, lead to practically identical positions of the amplitude poles in 
the complex mass plane. The amplitude has five poles:

$$
\begin{array}{cl}
\text { Resonance } & \text { Pole position }(\text { in } M e V) \\
& \\
f_{0}(980) & 1015 \pm 15-i(43 \pm 8) \\
f_{0}(1300) & 1300 \pm 20-i(120 \pm 20) \\
f_{0}(1500) & 1499 \pm 8-i(65 \pm 10) \\
f_{0}(1750) & 1750 \pm 30-i(125 \pm 70) \\
f_{0}\left(1530_{-250}^{+90}\right) & 1530_{-250}^{+90}-i(560 \pm 140) .
\end{array}
$$

The broad resonance $f_{0}\left(1530_{-250}^{+90}\right)$ is not a new object in meson physics: this is that one which was called $\epsilon(1300)$. A large width of $f_{0}\left(1530_{-250}^{+90}\right)$ is due to the accumulation of widths of neighbouring resonances.

Reliable and unambigous identification of the scalar glueball must be based upon the complete reconstruction of the multiplets $1^{3} P_{0} q \bar{q}$ and $2^{3} P_{0} q \bar{q}$. Each of these nonets consists of two scalar/isoscalar states $f_{0}$, one isovector/scalar state $a_{0}$, and the scalar kaon $K_{0}$. As was stated above, it is reasonable to perform the nonet classification of highly-excited $q \bar{q}$-states in terms of bare states, which do not contain clouds of real mesons. The analysis [30] fixed four $f_{0}^{b a r e}$ mesons, which are necessary for the construction of two nonets; the two lightest isotriplet resonances, $a_{0}(980)$ and $a_{0}(1450)$, are also known, see [9]. A full $K$-matrix analysis of the $10^{++}$wave, [36], provided the following resonance masses:

$$
a_{0}(980) \rightarrow(988 \pm 6)-i(46 \pm 10) \mathrm{MeV}, a_{0}(1450) \rightarrow(1565 \pm 30)-i(146 \pm 20) \mathrm{MeV} .
$$

It should be pointed out that in the PDG compilation [9] the mass of the second resonance is too low by about $100 \mathrm{MeV}$.

Corresponding bare states are as follows:

$$
a_{0}^{\text {bare }}(964 \pm 16), \quad a_{0}^{\text {bare }}(1670 \pm 70) .
$$

Identification of scalar resonances as members of the $1^{3} P_{0} q \bar{q}$ and $2^{3} P_{0} q \bar{q}$ always raised problems. Namely, according to [9, 37], the masses of the two lightest kaons are $1429 \pm 4 \pm 5 \mathrm{MeV}$ and $1945 \pm 10 \pm 20$ $\mathrm{MeV}$; these are noticeably higher than the average masses of other mesons which are candidates for the scalar-nonet members. This high position on the mass scale of the scalar kaon, $K_{0}(1430)$, gave impetus to models where the basic $1^{3} P_{0} q \bar{q}$-multiplet was fixed in the region $1350-1500 \mathrm{MeV}$, and the resonances $f_{0}(980)$ and $a_{0}(980)$ were considered as exotic states - hadron molecules [38], multiquark bags [39], or minions 40, 41].

In Ref. [42] the $K$-matrix re-analysis of the $S$-wave $K \pi$-spectrum was carried out to determine the $K_{0}^{\text {bare }}$. Another reason to re-analyse it was as follows. In Ref. [37] the $K \pi$-spectra have been investigated in two separate mass regions, $820-1580 \mathrm{MeV}$ and $1780-2180 \mathrm{MeV}$, but the mass region $1580-1780 \mathrm{MeV}$ was not included into the analysis of the $K \pi$ amplitude. Our experience in fitting the $00^{++}$-wave [30] teaches us that separate consideration of different mass regions leads to the loss of certain information. In order to get a full picture, a simultaneous fit is needed; moreover, at 1580-1780 $\mathrm{MeV}$ there is a rapid change of the amplitude.

As follows from the $K$-matrix fit of the $\left(I J^{P}=\frac{1}{2} 0^{+}\right)$wave [42], for a good description of the $K \pi$-spectrum in the region $800-2000 \mathrm{MeV}$ at least two $K_{0}$-states are necessary. Correspondingly, the $\frac{1}{2} 0^{+}$-amplitude of this minimal solution has poles near the physical region on the 2 nd sheet (under the $K \pi$-cut) and on the 3rd sheet (under $K \pi$ - and $K \eta^{\prime}$-cuts), at the following complex masses:

$$
(1415 \pm 30)-i(165 \pm 25) \mathrm{MeV}, \quad(1820 \pm 40)-i(125 \pm 35) \mathrm{MeV} .
$$

The $K \eta^{\prime}$ threshold, being in the vicinity of the resonance (at $1458 \mathrm{MeV}$ ), strongly influences the $\frac{1}{2} 0^{+}$amplitude, so the lowest $K_{0}$-state has a second pole which is located above the $K \eta^{\prime}$-cut, at 
$M=(1525 \pm 125)-i(420 \pm 80) \mathrm{MeV}$ : the situation is analogous to that observed for the $f_{0}(980)$ meson, which also has a two-pole structure of the amplitude due to the $K \bar{K}$-threshold. The $K \eta$ channel weakly influences the $\frac{1}{2} 0^{+} K \pi$ amplitude: experimental data [37] prove it as well as the quark combinatoric rules.

The minimal solution contains two $K_{0}^{\text {bare }}$ states:

$$
K_{0}^{\text {bare }}\left(1200_{-110}^{+60}\right), \quad K_{0}^{\text {bare }}\left(1820_{-75}^{+40}\right) .
$$

In this minimal solution, the lightest scalar bare kaon appears $200 \mathrm{MeV}$ lower than the amplitude pole, and this latter circumstance makes it easier to build the basic scalar nonet, with masses in the range $900-1200 \mathrm{MeV}$.

It is worth noting that the $K \pi$ spectra also allow solutions with three poles, with much better $\chi^{2}$; still, for these solutions the lightest kaon state, $K_{0}^{\text {bare }}$, does not leave the range $900-1200 \mathrm{MeV}$. In the tree-pole solution

$$
K_{0}^{\text {bare }}(1090 \pm 40), \quad K_{0}^{\text {bare }}\left(1375_{-40}^{+125}\right), \quad K_{0}^{\text {bare }}\left(1950_{-20}^{+70}\right),
$$

and the $K \pi$-amplitude has the poles:

$$
\begin{array}{rcc}
\text { II sheet } & M=998 \pm 15-i(80 \pm 15) & \mathrm{MeV} \\
\text { II sheet } & M=1426 \pm 15-i(182 \pm 15) & \mathrm{MeV} \\
\text { III sheet } & M=1468 \pm 30-i(309 \pm 15) & \mathrm{MeV} \\
\text { III sheet } & M=1815 \pm 25-i(130 \pm 25) & \mathrm{MeV} .
\end{array}
$$

The state $K_{0}^{\text {bare }}\left(1375_{-40}^{+125}\right)$, being near the $K \eta^{\prime}$ threshold, results in doubling the amplitude poles around $1400 \mathrm{MeV}$. It should be underlined that masses of the lightest bare kaon states obtained by the two- and three-pole solutions coincide within the errors.

The $K$-matrix fit of the $\frac{1}{2} 0^{+}$wave makes it possible to complete, in terms of bare states, the construction of the two lowest scalar nonets. In line with the result for the $00^{++}$wave [30], where two solutions for an extra state (candidate for the glueball) were found, there are two variants for the nonet classification of scalar mesons. The basic $1^{3} P_{0} q \bar{q}-$ nonet is the same for both variants:

$$
a_{0}^{\text {bare }}(960 \pm 30), f_{0}^{\text {bare }}(720 \pm 100), f_{0}^{\text {bare }}\left(1260_{-30}^{+100}\right), K_{0}^{\text {bare }}\left(1200_{-150}^{+90}\right) .
$$

It should be particularly stressed that the wave function $f_{0}^{\text {bare }}(720)$ in the flavour space is close to the octet one; indeed, $\phi(720)=-69^{\circ} \pm 12^{\circ}$, while $\phi_{\text {octet }}=-54.7^{\circ}$. Correspondingly, $f_{0}^{\text {bare }}(1260)$ is close to the flavour singlet. A similar situation is observed in the pseudoscalar sector where the flavour wave functions $\eta$ and $\eta^{\prime}$ are close to the octet and singlet ones. It is even more analogous, if one takes into consideration that the mass diffrence of isoscalar states in these sectors coincide with each other, and the scalar masses are not much larger than corresponding masses of pseudoscalars, $m_{\text {scalar }}-m_{\text {pseudoscalar }} \simeq(200 \pm 100) \mathrm{MeV}$. Such coincidences clearly point towards parity degeneration of the interaction forces in isoscalar channels.

Thus, one may conclude: the basic nonet of scalar mesons is uniquely fixed by the $K$-matrix fit of meson sspectra. It is located rather low on the mass scale, in the range 750-1250 MeV. Here, at mass values below $1200 \mathrm{MeV}$, there is no room for exotic states.

The $2^{3} P_{0} q \bar{q}$ nonet contains the following states in Solution I:

$$
\begin{gathered}
a_{0}^{\text {bare }}(1640 \pm 40), f_{0}^{\text {bare }}(1600 \pm 50), f_{0}^{\text {bare }}\left(1810_{-100}^{+30}\right), \\
K_{0}^{\text {bare }}\left(1375_{-40}^{+125}\right) \text { or } K_{0}^{\text {bare }}\left(1820_{-60}^{+40}\right) .
\end{gathered}
$$


An extra state with respect to the nonet classification is $f_{0}^{\text {bare }}\left(1235_{-30}^{+150}\right)$.

In Solution II the $2^{3} P_{0} q \bar{q}$ nonet looks like:

$$
\begin{gathered}
a_{0}^{\text {bare }}(1640 \pm 40), f_{0}^{\text {bare }}\left(1235_{-30}^{+150}\right), f_{0}^{\text {bare }}\left(1810_{-100}^{+30}\right), \\
K_{0}^{\text {bare }}\left(1375_{-40}^{+125}\right) \text { or } K_{0}^{\text {bare }}\left(1820_{-60}^{+40}\right) .
\end{gathered}
$$

In this solution an extra state is $f_{0}^{\text {bare }}(1600 \pm 50)$; once again it should be stressed that the mass of this state appears just in the mass region where Lattice calculations for the mass of the lightest scalar gluonium point; also the coupling constants with meson channels agree with the quark combinatorics ratios for the gluonium decay.

Immediately after performing the $K$-matrix analysis in the range up to $1900 \mathrm{MeV}$, the problem of presentation of the $00^{++}$amplitude as a dispersion integral has been raised. The dispersion $N / D$ representation correctly restores analytic properties of partial amplitude in the whole complex $s$ plane. In addition, and this the principal point, within the dispersion representation it is possible to reconstruct the propagator matrix, thus evaluating the mixing of the $q \bar{q}$-states and the glueball, and then to restore correctly the gluonium mass. The dispersive $N / D$-description of the wave $00^{++}$has been performed in Refs. [6, 7]: in Ref. [6] the region 1200-1700 MeV where three scalar/isoscalar states are located, has been studied; then, in Ref. [7] the region under investigation has been extended to $1900 \mathrm{MeV}$, with the fourth state, $f_{0}(1780)$, included into consideration.

The results of the $N / D$-representation of the $00^{++}$wave allowed us to draw the picture of mixing for the lowest scalar gluonium: it mixes with the two neighbouring $q \bar{q}$ states - members of the multiplets $1^{3} P_{0}$ and $2^{3} P_{0}$, and the resonance- descendant of a pure glueball accumulates large parts of the widths of the neighbouring resonances, being transformed into the broad state $f_{0}\left(1530_{-250}^{+90}\right)$.

It should be emphasized that the state $f_{0}^{\text {bare }}$, which was found in the $K$-matrix fit, does not explicitly describe the gluodynamic glueball, for the state $f_{0}^{\text {bare }}$ contains nongluonic degrees of freedom related to real parts of the loop diagrams (imaginary parts are responsible for the decay process). The dispersion relation $N / D$ method is able to restore the real and imaginary parts of the loop diagrams, thus providing a complete picture of mixing, so it also restores the mass of gluonium. In Solution I it is equal to

$$
m_{\text {gluonium }}=1225 \mathrm{MeV} \text {, }
$$

and in Solution II:

$$
m_{\text {gluonium }}=1633 \mathrm{MeV} \text {. }
$$

The mass $m_{\text {gluonium }}=1633 \mathrm{MeV}$ (Solution II) agrees well with the mass values of the lightest scalar glueball obtained in Lattice calculations.

It is rather striking that both solutions obtained in the dispersive technique provide practically the same structure for the $00^{++}$wave and the quark-gluon content of physical resonances: in both solutions the broad resonance, $f_{0}\left(1530_{-250}^{+90}\right)$, is a descendant of the gluonium, keeping about $40-50 \%$ of its component, while the remnant part of the gluonium is shared between $f_{0}(1300)$ and $f_{0}(1500)$. From this point of view, the structure of resonances in the range $1300-1600 \mathrm{MeV}$ is uniquely solved.

The formation of the broad state which is seen in the $00^{++}$wave raises a question about the presence of such effects in other waves as well, for it is reasonable to believe that exotic mesons (glueballs and hybrids) with other quantum numbers can also afford a width accumulation. Because of that, the search for other exotic mesons must be inseparably linked with the study of broad resonances.

There appears another problem which is not less intriguing: the broad resonance, after having absorbed the widths of its neighbouring resonances, plays the role of a locking state, since it prevents the decay of neighbouring states with the same quantum numbers. This means that the broad resonance actually plays the role of a dynamic barrier for the nearby states. How does this dynamic barrier relate to the confinement barrier? - Only detailed investigations of broad resonances in other waves could answer this question. 


\section{$2 \quad K$-matrix and the dispersive $N / D$-representation of the scat- tering amplitude}

In this Section a brief review is done for the technique used in the analysis of meson spectra. Namely, analytic properties of the amplitude are discussed together with the connection of the dispersion $N / D$ representation to the $K$-matrix approach. The role of short and large distances in the formation of meson spectra under investigation is also discussed, and in this connection a notion "bare state" is introduced. The quark combinatoric relations between the decay couplings of the glueball to meson channels are considered in comparison with similar relations for $q \bar{q}$-states: these relations provide the basis for the nonet systematics of mesons.

\subsection{Scattering amplitude, $T$-matrix and $K$-matrix}

Basing on a simple example, let us get over the terminology and notations used for the analysis of meson spectra.

In terms of the wave function, which expresses relative movement of two spinless particles, the scattering at large distances is described by the incoming plane and outgoing spherical waves, with the coefficient $f(\theta)$ related to the partial amplitudes as follows:

$$
f(\theta)=\frac{1}{2 i k} \sum_{\ell=0}^{\infty}(2 \ell+1) P_{\ell}(\cos \theta)\left[e^{2 i \delta_{\ell}(k)}-1\right] .
$$

This formula is written for the one-channel scattering in absence of absorbtion ( $k$ is the momentum of relative movement, $\ell$ is the angular momentum and $\theta$ is the scattering angle). The $T$-matrix element is determined by the scattering phase shift $\delta_{\ell}$ :

$$
T_{\ell}=\frac{1}{2 i}\left[e^{2 i \delta_{\ell}}-1\right]=e^{i \delta_{\ell}} \sin \delta_{\ell} .
$$

For the investigation of analytic properties, it is suitable to use an amplitude with other normalization:

$$
A_{\ell}=\frac{1}{2 i \rho(k)}\left[e^{2 i \delta_{\ell}}-1\right]
$$

where $\rho(k)$ is the invariant two-particle phase space factor:

$$
\rho(k)=\int d \Phi\left(P ; k_{1}, k_{2}\right), \quad d \Phi\left(P ; k_{1}, k_{2}\right)=\frac{1}{2} \frac{d^{3} k_{1}}{(2 \pi)^{3} 2 k_{10}} \frac{d^{3} k_{2}}{(2 \pi)^{3} 2 k_{20}}(2 \pi)^{4} \delta^{(4)}\left(P-k_{1}-k_{2}\right) .
$$

Invariant phase space factor is determined by three four-momenta: the total momentum of scatterred particles, $P$, with $P^{2}=s$, and the momenta of particles 1 and $2, k_{1}$ and $k_{2}$, respectively. For equal masses of particles 1 and 2 , we get:

$$
\rho(k)=\frac{k}{8 \pi \sqrt{s}}, \quad k=\sqrt{\frac{s}{4}-m^{2}} .
$$

The $K$-matrix representation of the amplitude $A_{\ell}$ reads:

$$
A_{\ell}=\frac{K_{\ell}\left(k^{2}\right)}{1-i \rho(k) K_{\ell}\left(k^{2}\right)} .
$$

$K_{\ell}$ is real in the physical region; the imaginary part of the amplitude is explicitly written in Eq. (2.6). In addition, $K_{\ell}$ as a function of the invariant energy squared $s$ is analytic near the threshold 
singularity, $s=4 m^{2}$ : a singular term is singled out, it is explicitly given by the two-particle phase space factor $\rho$.

In presence of absorbtion, the scattering with an absorbtion is described by the absorbtion coefficient $\eta_{\ell}$ inserted to the partial wave expansion (2.1):

$$
\left[e^{2 i \delta_{\ell}}-1\right] \rightarrow\left[\eta_{\ell} e^{2 i \delta_{\ell}}-1\right]
$$

Here $0 \leq \eta_{\ell} \leq 1$; the case $\eta_{\ell}=0$ corresponds to full absorbtion.

It is suitable to display the energy-dependent amplitude $T_{\ell}$ on the Argand-diagram, which is an appropriate instrument for searching resonances. The $T$-matrix element at fixed $k$ (or $s$ ) corresponds to the point on the plane $\left(\operatorname{Re} T_{\ell}, \operatorname{Im} T_{\ell}\right)$. As a function of $k$, it draws a trajectory on the circle with the radius equal to $1 / 2$ and the centre in the point $0, i / 2$. In the inelasticity case, the trajectory $T_{\ell}$ enters the internal part of the circle.

The $K$-matrix representation of the amplitude with an absorbtion requires fixing inelastic channels. Consider the inelasticity occurring due to another two-particle channel; we denote these channels by indices 1 and 2. Then the elastic scattering amplitude $1+1^{\prime} \rightarrow 1+1^{\prime}$ (denoted as $A_{11}$, index $\ell$ is omitted) can be presented in the form of Eq. (2.6):

$$
A_{11}=\frac{K\left(k^{2}\right)}{1-i \rho_{1} K\left(k^{2}\right)} .
$$

However, the block $K\left(k^{2}\right)$ has an imaginary part above the threshold of the second channel:

$$
K\left(k^{2}\right)=K_{11}+i \frac{K_{12} \rho_{2} K_{21}}{1-i \rho_{2} K_{22}} .
$$

Here $\rho_{2}$ is phase space factor of the second channel $2+2^{\prime}$, and matrix elements $K_{11}, K_{12}=K_{21}$ and $K_{22}$ are real functions of $k^{2}$ in the physical region. Threshold singularities of the channels 1 and 2, which are located at $s=\left(m_{1}+m_{1}^{\prime}\right)^{2}$ (threshold of the channel 1) and at $s=\left(m_{2}+m_{2}^{\prime}\right)^{2}$ (threshold of the channel 2), are explicitly written in (2.8) and (2.9) - they are present in the phase space factors $\rho_{1}$ and $\rho_{2}$, respectively. The function $K\left(k^{2}\right)$ is real below the threshold of the second channel, $\left(m_{1}+m_{1}^{\prime}\right)^{2}<s<\left(m_{2}+m_{2}^{\prime}\right)^{2}$, because in this region $\rho_{2}=i\left|\rho_{2}\right|$.

\subsection{Dispersion relation $N / D$-method and the $K$-matrix representation}

The dispersion relation $N / D$ method [43] correctly reproduces analytic properties of the amplitude on the whole $s$-plane. Following [44] 45], we present here the elements of this method which are used in the analysis of meson spectra.

Partial amplitude $A(s)$ (as before, the index $\ell$ is omitted for brevity) is written in form of the ratio

$$
A(s)=\frac{N(s)}{D(s)} .
$$

$N(s)$ is a function of the complex variable $s$. It has only left-hand side singularities of the amplitude, which are related to the interaction forces, i.e. to the diagrams with meson exchanges in the crossing channels (see Fig. 1). These singularities are located on the left from the threshold singularities, at $s=\left(m_{1}+m_{1}^{\prime}\right)^{2}-m_{\text {crossing }}^{2}$.

The $D$-function contains only right-hand side singularities, which result from the rescattering of particles in the $s$-channel. Figure 2.1 shows the corresponding rescattering processes.

First, we consider the one-channel case, with equal particle masses $m_{1}=m_{1}^{\prime}$, in this case the $D$-function assumes the following form:

$$
D(s)=1-B(s), \quad B(s)=\int_{4 m^{2}}^{\infty} \frac{d s^{\prime}}{\pi} \frac{N\left(s^{\prime}\right) \rho\left(s^{\prime}\right)}{s^{\prime}-s-i 0} .
$$


Here index 1 is omitted: $m_{1} \rightarrow m, \rho_{1} \rightarrow \rho$. The form of equation (2.11) suggests that $D(s) \rightarrow 1$ at $s \rightarrow \infty$ (more generally, $D(s) \rightarrow$ Const at $s \rightarrow \infty$, since this case can be reduced to Eq. (2.11) by the re-definition of $N(s)$ ). Moreover, in Eq. (2.11) it is also suggested that the $D$-function does not contain the Castillejo-Dalitz-Dyson poles (a detailed description of the $N / D$-method may be found in Refs. 43, 44).

Representation of the $N$-function in the form of a sum of the separable vertex functions 44 is likely to be a reasonable anzatz: this technique is successfully applied for the description of the $N N$ scattering amplitude 45; in addition, the technique is elaborated for the presentation of the $t$-channel exchange diagrams as a sum of separable vertex functions 46 . For the simplest case, which is discussed below, $N(s)=g^{2}(s)$. Then

$$
A(s)=\frac{g^{2}(s)}{1-B(s)}, \quad B(s)=\int_{4 m^{2}}^{\infty} \frac{d s^{\prime}}{\pi} \frac{g\left(s^{\prime}\right) \rho\left(s^{\prime}\right) g\left(s^{\prime}\right)}{s^{\prime}-s-i 0} .
$$

Expanding Eq. (2.12) in a series with respect to $B(s)$, we represent the amplitude $A(s)$ as a sum of diagrams shown in Figs. 2a, 2b, 2c, etc.: $B(s)$ in Eq. (2.12) is a loop diagram. At $s>4 m^{2}$, the loop diagram is a complex quantity:

$$
\operatorname{ImB}(S)=g^{2}(s) \rho(s), \quad \operatorname{Re} B(s)=P \int_{4 m^{2}}^{\infty} \frac{d\left(s^{\prime}\right)}{\pi} \frac{g^{2}\left(s^{\prime}\right) \rho\left(s^{\prime}\right)}{s^{\prime}-s} .
$$

The amplitude (2.12) stands for the case when the partial wave amplitude does not contain input particles: the bound states, if any, are formed by particle interaction taken in the $N$-function. The inclusion of input particles into the amplitude corresponds to the assumption on $D(s)$ increasing at $s \rightarrow \infty$. The linearly growing $D(s)$ can be written in the form:

$$
D(s)=m_{0}^{2}-s-B(s), \quad B(s)=\int_{4 m^{2}}^{\infty} \frac{d\left(s^{\prime}\right)}{\pi} \frac{g^{2}\left(s^{\prime}\right) \rho\left(s^{\prime}\right)}{s^{\prime}-s-i 0} .
$$

The amplitude

$$
A(s)=\frac{g^{2}(s)}{m_{0}^{2}-s-B(s)}
$$

is an infinite set of diagrams shown in Figs. 2d, 2e, 2f, etc.; $B(s)$ stands for the loop diagram and $\left(m_{0}^{2}-s\right)^{-1}$ is the propagator of the input particle.

The $K$-matrix representation of the $A(s)$ amplitude is related to the explicit separation of the imaginary part of the loop diagram:

$$
A(s)=\frac{g^{2}(s)}{m_{0}^{2}-s-\operatorname{Re} B(s)-i \rho(s) g^{2}(s)}=\frac{K(s)}{1-i \rho(s) K(s)}, \quad K(s)=\frac{g^{2}(s)}{m_{0}^{2}-s-\operatorname{Re} B(s)} .
$$

In the two-particle loop diagram, the function $\operatorname{Re} B(s)$ is analytical at the point $s=4 m^{2}$. This means that poles are the only singulariries of $K(s)$ in the physical region. However, at the left half-plane $s$, $K(s)$ has singulariries related to the $t$-channel exchanges.

The poles of the amplitude $A(s)$, which are determined by the condition

$$
m_{0}^{2}-s-B(s)=0
$$

are related to particles with quantum numbers of the partial wave under consideration. If the pole is above the threshold, at $\operatorname{Re} s>4 m^{2}$, we deal with the resonance; this very case is studied further. Let the equality (2.17) be satisfied in the point:

$$
s=M^{2} \equiv \mu^{2}-i \Gamma \mu .
$$


Expanding the real part of the denominator (2.15) in a series near $s=\mu^{2}$, one has:

$$
m_{0}^{2}-s-\operatorname{ReB}(s) \simeq\left(1+\operatorname{Re}^{\prime}\left(\mu^{2}\right)\right)\left(\mu^{2}-s\right)-i g^{2}(s) \rho(s) .
$$

The standard Breit-Wigner approximation comes when $\operatorname{Im} B(s)$ is fixed in the point $s=\mu^{2}$. If the pole is located not far from the threshold singularity $s=4 m^{2}$, it is necessary to keep the $s$-dependence in the phase space factor, and we use the modified Breit-Wigner formula:

$$
A(s)=\frac{\gamma}{\mu^{2}-s-i \gamma \rho(s)}, \quad \gamma=\frac{g^{2}\left(\mu^{2}\right)}{1+\operatorname{Re} B^{\prime}\left(\mu^{2}\right)} .
$$

Similar resonance approximation may be also carried out for the $K$-matrix amplitude representation, that corresponds to the expansion for $K(s)$ given by Eq. (2.16) near the point $s=\mu^{2}$ :

$$
K(s)=\frac{g^{2}(K)}{\mu^{2}-s}+f .
$$

Here

$$
g^{2}(K)=\frac{g^{2}\left(\mu^{2}\right)}{1+\operatorname{Re} B^{\prime}\left(\mu^{2}\right)}, \quad f=\frac{g^{2}\left(\mu^{2}\right)}{2\left(1+\operatorname{Re} B^{\prime}\left(\mu^{2}\right)\right)}-\frac{2 g\left(\mu^{2}\right) g^{\prime}\left(\mu^{2}\right)}{1+\operatorname{Re} B^{\prime}\left(\mu^{2}\right)}
$$

\subsection{Multichannel scattering}

The resonance amplitude (2.15) can be easily generalized for the $n$ channel case. The corresponding transition amplitude $b \rightarrow a$ is equal to:

$$
A_{a b}(s)=\frac{g_{a}(s) g_{b}(s)}{m_{0}^{2}-s-B(s)}, \quad B(s)=\sum_{c=1}^{n} B_{c c}(s),
$$

where $B_{c c}$ is defined by the standard expression (see Eq. (2.14)), with the properly chosen phase space factor, vertex function and integration region:

$$
g^{2}\left(s^{\prime}\right) \rho\left(s^{\prime}\right) \rightarrow g_{c}^{2}\left(s^{\prime}\right) \rho_{c}\left(s^{\prime}\right), \quad 4 m^{2} \rightarrow 4 m_{c}^{2}
$$

The transition amplitudes $A_{a b}$ form a matrix $\hat{A}$. The amplitude written in the $K$-matrix representation reads:

$$
\hat{A}=\hat{K} \frac{I}{I-i \hat{\rho} \hat{K}}
$$

where $\hat{K}$ is the $n \times n$ matrix, $K_{a b}(s)=K_{b a}(s), I$ is the unit $n \times n$ matrix $I=\operatorname{diag}(1,1, \ldots, 1)$, and $\hat{\rho}$ is the diagonal matrix of phase space factors:

$$
\hat{\rho}=\operatorname{diag}\left(\rho_{1}(s), \rho_{2}(s), \ldots, \rho_{n}(s)\right) .
$$

The $K$-matrix elements are equal to:

$$
K_{a b}(s)=\frac{g_{a}(s) g_{b}(s)}{m_{0}^{2}-s-\operatorname{Re} B(s)} .
$$

In the vicinity of the resonance, the $K$-matrix elements may be expanded in a series: in this case we have a representation of the $K$-matrix elements similar to that of Eq. (2.21). 


\section{$2.4 q \bar{q}$-mesons: the problem of small and large distances}

The $q \bar{q}$ classification of meson states in the vicinity of $1000-2000 \mathrm{MeV}$ faces the problem of the quark-hadron duality as well as a tightly related problem of separating large- and small- $r$ interactions contributing into formation of meson spectra.

Let us discuss these problems using the language of the standard quark model. In this model the $q \bar{q}$ levels are determined by the potential which increases infinitely with $r: V(r) \sim \alpha r$ (see Fig. $3 \mathrm{a})$. Infinitely rising potential creates an infinite set of the $q \bar{q}$ levels. However, it is obvious that the standard quark model is a simplified picture, since only the lowest $q \bar{q}$-levels are stable with respect to hadron decays. The heavier states are decaying by hadron channels: an excited $(q \bar{q})_{a}$-state produces new $q \bar{q}$ pair, then the $(q \bar{q})_{a}+(q \bar{q})$ quarks recombine into mesons, which leave the confinement trap with a formation of the continuous meson spectrum. This structure is conventionally shown in Fig. $3 \mathrm{~b}$, where the interaction related to the confinement is shown as a certain potential barrier, namely, the interaction at $r<R_{\text {confinement }}$ creates the discrete levels of $q \bar{q}$ spectra, while the transitions

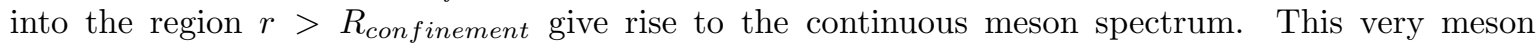
spectrum is observed in the experiment. This problem of reconstruction of the $q \bar{q}$-levels created at $r<R_{\text {confinement }}$ is directly related to the determination of the effect of the meson decay spectra on the level shift: classification of \# levels requires elimination of the components of real mesons that are the decay products of real meson.

The $K$-matrix representation of the amplitude resolves the problem of excluding the components of real mesons; formally, it corresponds to the limit $\rho_{a} \rightarrow 0$ in Eq. (2.25). If only leading pole singularities are taken into account, the transition amplitude $b \rightarrow a$ assumes the form:

$$
A_{a b}^{\mathrm{bare}}(s)=K_{a b}(s)=\frac{g_{a}(K) g_{b}(K)}{\mu^{2}-s}+f_{a b} .
$$

Thus, the $K$-matrix pole corresponds to the state with the removed cloud of real mesons. For this reason, we call corresponding states "bare mesons" 29, 30]. However, one should distinguish between this notation and that of "bare particles" of field theory, where a cloud includes virtual mass-off-shell particles as well.

In the case when the $q \bar{q}$-spectrum contains several states with the same quantum numbers, the amplitude $A_{a b}^{\text {bare }}(s)$ is determined by the sum of the corresponging poles:

$$
A_{a b}^{\mathrm{bare}}(s)=\sum_{\alpha} \frac{g_{a}^{(\alpha)}(K) g_{b}^{(\alpha)}(K)}{\mu_{\alpha}^{2}-s}+f_{a b} .
$$

Representation of the amplitude responsible for the interaction at $r<R_{\text {confinement }}$ in the form of a series of poles is not new: it was widely used in dual models for contributions which are leading in the $1 / N_{c}$-expansion. From the point of view of these models, the $s$-independent term $f_{a b}$ is the thorough contribution of poles which are distant from the region under consideration.

Coupling constants of the bare state, $g_{a}^{(\alpha)}(K)$, are a source of information on the quark-gluon content of this state.

\subsection{Coupling constants of the gluonium and $q \bar{q}$-states to meson channels: the rules of the $1 / N$-expansion and quark combinatoric relations}

Quark-gluon content of states related to the $K$-matrix poles (bare states) is revealed in relations between couplings of these poles to meson channels, $g_{a}^{(\alpha)}$.

First, let us evaluate these couplings using the rules of $1 / N$-expansion; this evaluation will be done both for the transitions glueball $\rightarrow$ two mesons and for the transitions $q \bar{q}-$ state $\rightarrow$ two mesons. 
For this purpose, we consider the gluon loop diagram which corresponds to the two-gluon self-energy part: glueball $\rightarrow$ two gluons $\rightarrow$ glueball (see Fig. 2.4a). This loop diagram is of the order of unity, provided the glueball is a two-gluon composite particle: $B(G \rightarrow g g \rightarrow G) \sim g_{G \rightarrow g g}^{2} N_{c}^{2} \sim 1$, where $g_{G \rightarrow g g}$ is a coupling constant of a glueball to two gluons. Therefore,

$$
g_{G \rightarrow g g} \sim 1 / N_{c} .
$$

Coupling constant for the transition $g_{G \rightarrow q \bar{q}}$ is determined by the diagrams of Fig. 4b. Similar evaluation gives:

$$
g_{G \rightarrow q \bar{q}} \sim g_{G \rightarrow g g} g_{Q C D}^{2} N_{c} \sim 1 / N_{c} .
$$

Here $g_{Q C D}$ is the quark-gluon coupling constant, which is of the order of $1 / \sqrt{N_{c}}$. 8 . Coupling constant for the transition glueball $\rightarrow$ two mesons in the leading $1 / N_{c}$ terms is governed by the diagrams of Fig. 4c:

$$
g_{G \rightarrow m m}^{L} \sim g_{G \rightarrow q \bar{q}} g_{m \rightarrow q \bar{q}}^{2} N_{c} \sim 1 / N_{c} .
$$

Here the approximate equality $g_{m \rightarrow q \bar{q}} \sim 1 / \sqrt{N_{c}}$ is used which follows from the fact that the loop diagram of the meson propagator (see Fig. 4d) is of the order of the unity: $B(m \rightarrow q \bar{q} \rightarrow m) \sim$ $g_{m \rightarrow q \bar{q}}^{2} N_{c} \sim 1$. The diagram of Fig. 2.4e-type governs the couplings for the transition glueball $\rightarrow$ two mesons in the next-to-leading terms of the $1 / N_{c^{-}}$expansion:

$$
g_{G \rightarrow m m}^{N L} \sim g_{G \rightarrow g g} g_{Q C D}^{4} g_{m \rightarrow q \bar{q}}^{2} N_{c}^{2} \sim 1 / N_{c}^{2} .
$$

As was mentioned above, the glueball can decay into channels (1.8). In this way, the production of light quarks by gluons is going on with a violation of the flavour blindness, $u \bar{u}: d \bar{d}: s \bar{s}=1: 1: \lambda$. Within such an assumption, the coupling constants for the transition glueball $\rightarrow$ two pseudoscalar mesons can be calculated using quark combinatorics. The rules of quark combinatorics were successfully applied to the calculation of yields of secondary particles in the hadron-hadron high energy collisions 477 and to the decay $J / \psi \rightarrow$ hadrons [48. The calculation of couplings in the decays glueball $\rightarrow$ mesons has been carried out in Refs. [20, 34, 49].

The glueball decay couplings to channels (1.8) are given in Table I for leading, $g_{G \rightarrow m m}^{L}$, and nextto-leading, $g_{G \rightarrow m m}^{N L}$, terms of the $1 / N$-expansion. Unknown dynamics of the decay is hidden in the parameters $G_{L}$ and $G_{N L}$. The decay constant to the channel $n$ is a sum of both contributions:

$$
g_{G \rightarrow m m}^{L}(n)+g_{G \rightarrow m m}^{N L}(n) .
$$

The second term is suppressed, as compared to the first one, by the factor $N_{c}$; the experience in calculation of quark diagrams teaches us that this suppression is of the order of $1 / 10$.

The sum of couplings squared satisfies the sum rules:

$$
\sum_{n}\left(g_{G \rightarrow m m}^{L}(n)\right)^{2} I(n)=\frac{1}{2} G_{L}^{2}(2+\lambda)^{2}, \quad \sum_{n}\left(g_{G \rightarrow m m}^{N L}(n)\right)^{2} I(n)=\frac{1}{2} G_{N L}^{2}(2+\lambda)^{2},
$$

where $I(n)$ is an identity factor for the particles produced, see Table I. These sum rules follow from the quark-hadron duality: the sum of squared coupling constants over the whole set of flavour states is equivalent to the sum of cut diagrams with the quark loops (diagrams of the type of Fig. $4 \mathrm{f}$ for leading and of the type of Fig. $4 \mathrm{~g}$ for next-to-leading terms). Each quark loop contains the factor $(2+\lambda)$ related to the summation over light flavours, see Eq. (1.10).

Quark combinatoric rules may be applied to the calculation of couplings of $(q \bar{q})_{a}$-mesons to pseudoscalar channels (1.8). There exist two types of transitions $(q \bar{q})_{a}$-state $\rightarrow$ two mesons: they are shown in Fig. 5a and 2.5b. The type of process represented by the diagram of Fig. 5a is leading according to the rules of $1 / N$ expansion; its coupling constant is of the order of

$$
g_{m(a) \rightarrow m m}^{L} \sim g_{m \rightarrow q \bar{q}}^{3} N_{c} \sim \frac{1}{\sqrt{N_{c}}} .
$$


The decay constant for the process of Fig. 5b is of the order of

$$
g_{m(a) \rightarrow m m}^{N L} \sim g_{m \rightarrow q \bar{q}}^{3} N_{c}^{2} g_{m \rightarrow q \bar{q}}^{4} \sim \frac{1}{N_{c} \sqrt{N_{c}}} .
$$

The coupling constants for the decays $(q \bar{q})_{a} \rightarrow \pi \pi, K \bar{K}, \eta \eta, \eta \eta^{\prime}, \eta^{\prime} \eta^{\prime}$ are shown in Table 2 both for the leading and next-to-leading orders; $g^{L}$ and $g^{N L}$ are parameters, in which the unknown dynamics of soft decay is hidden. Concerning the glueball decay, the coupling for the $(q \bar{q})_{a}$-meson decay to the channel $n$ is the sum of both terms:

$$
g_{m(a) \rightarrow m m}^{L}(n)+g_{m(a) \rightarrow m m}^{N L}(n) .
$$

These two terms in Eq. (2.38) define the decay constant of the $(q \bar{q})_{a}$-meson in general case: different variants of fixing the ratios of coupling constants correspond to different choices of the ratio $g_{L} / g_{N L}$. The examples of different fixations of $g_{L} / g_{N L}$ may be found in 40, 49].

Let us stress once again that the coupling constant ratios for the $(q \bar{q})_{a}$-states (Table II) become identical to those of the glueball, when $\phi=\arctan \sqrt{\frac{\lambda}{2}}$ : it is valid for leading and next-to-leading contributions. Therefore, that, on the basis of a study of couplings to the hadron decay channels only, it is impossible to distinguish between the glueball and the $I=0(q \bar{q})_{a}$-meson with the mixing angle $\phi$ close to $30^{\circ}$. 


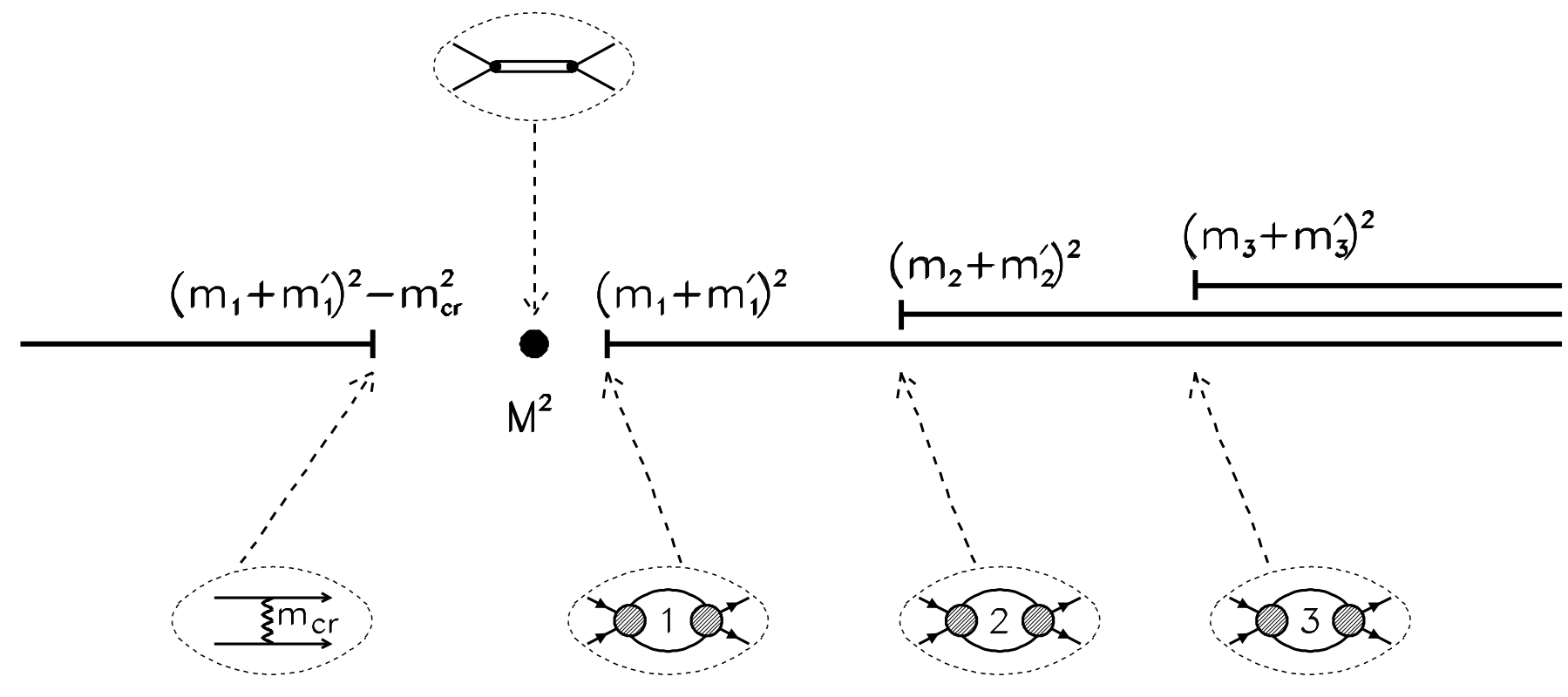

Fig. 1. Complex $s$-plane and positions of singularities of the partial amplitude: right-hand singularities at $s \geq\left(m_{1}+m_{1}^{\prime}\right)^{2}$ are due to the elastic and inelastic rescatterings, left-hand ones are due to the interaction forces, that is, particle exchanges in the crossing channels.

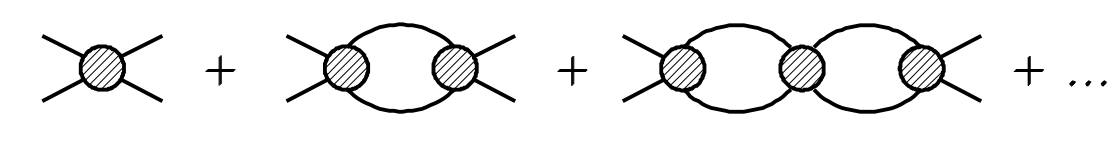

a

$\sigma$

B

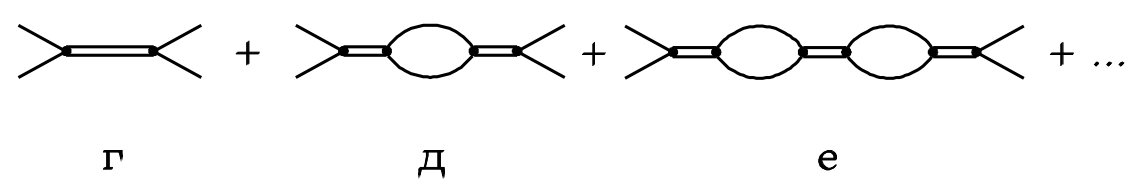

Fig. 2. Diagrams standing for the $s$-channel scattering. 

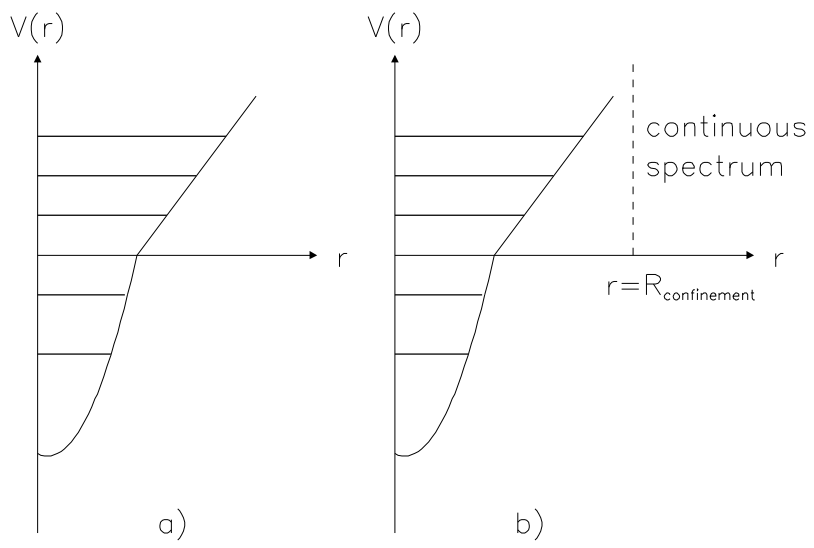

Fig. 3. a) Standard quark model potential with stable $q \bar{q}$-levels, b) Potential with the unstable highly excited levels which corresponds to the realistic situation for $q \bar{q}$ states. 


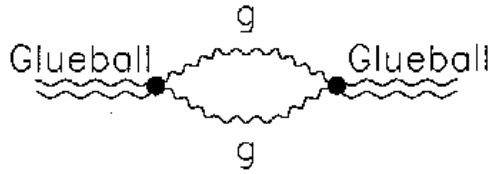

a)

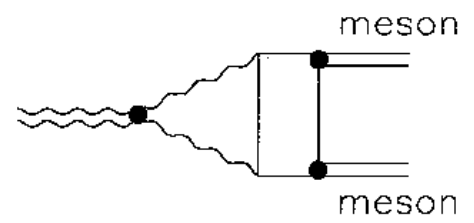

c)

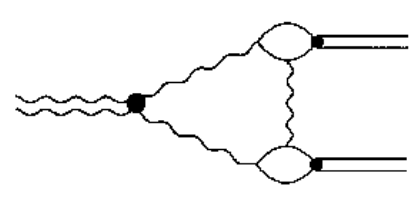

e)

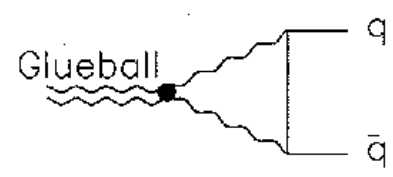

b)

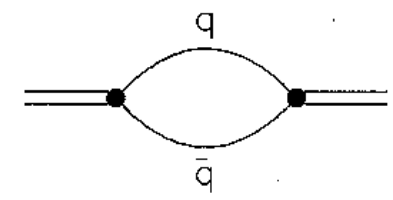

d)

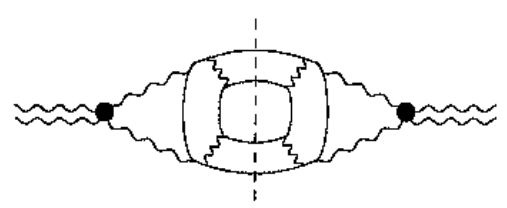

f)

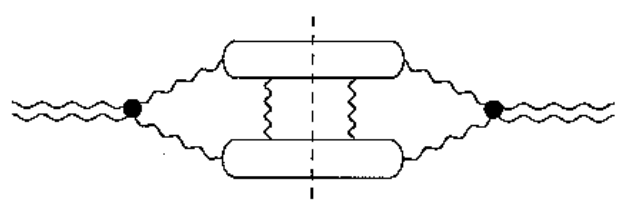

g)

Fig. 4. Diagrams for the glueball decay into two mesons.

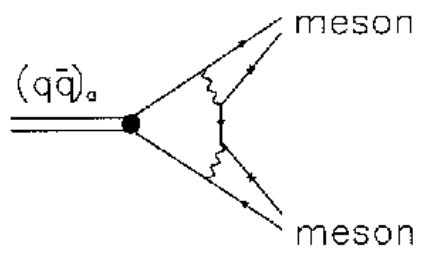

a)

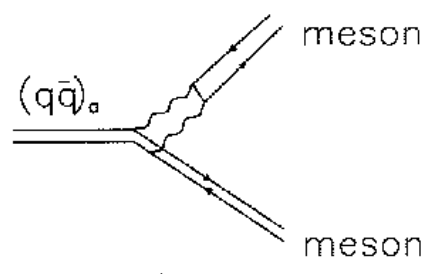

b)

Fig. 5. Diagrams for the decay of the $(q \bar{q})_{a}$-state into two mesons. 


\section{$3 \quad K$-matrix analysis of meson spectra and nonet classification of $q \bar{q}$-states}

In this Section, the results obtained in Ref. [30, 36, 42] for the waves $00^{++}, 10^{++} 02^{++}, 12^{++}$and $\frac{1}{2} 0^{+}$are presented. On the basis of this analysis, the nonet classification of $q \bar{q}$-states is established.

\section{$3.1 \quad K$-matrix fit of $00^{++}$-wave: the spectra $\pi \pi, K \bar{K}, \eta \eta$ and $\eta \eta^{\prime}$}

To describe the spectra, in Ref. [30] the standard $K$-matrix representation of the $00^{++}$amplitude (2.25) was used, where $K_{a b}$ is the $5 \times 5$ matrix $(a, b=1,2, \ldots, 5)$, with the following notations for channels: $1=\pi \pi, 2=K \bar{K}, 3=\eta \eta, 4=\eta \eta^{\prime}, 5=(\pi \pi \pi \pi+$ other multimeson states $)$.

Matrix elements of $K_{a b}$ are parametrised in the form similar to Eq. (2.28):

$$
K_{a b}=\left(\sum_{\alpha} \frac{g_{a}^{(\alpha)} g_{b}^{(\alpha)}}{M_{\alpha}^{2}-s}+f_{a b} \frac{1 \mathrm{GeV}^{2}+s_{0}}{s+s_{0}}\right),
$$

with the restriction $s_{0} \geq 1 \mathrm{GeV}^{2}$.

The following formulae provide the description of the spectra $\pi \pi, \eta \eta$ and $\eta \eta^{\prime}$, obtained by GAMS for the reactions with the $t$-channel pion exchange:

$$
\begin{gathered}
A_{\pi N \rightarrow N b}=g\left(\bar{\psi}_{N} \gamma_{5} \psi_{N}\right) F(t) D(t) K_{1 a}(t)(1-i \hat{\rho} \hat{K})_{a b}^{-1}, \\
K_{1 a}(t)=\left(\Sigma_{\alpha} \frac{g_{1}^{(\alpha)}(t) g_{a}^{(\alpha)}}{M_{\alpha}^{2}-s}+f_{1 a}(t) \frac{1 \mathrm{GeV}^{2}+s_{0}}{s+s_{0}}\right) .
\end{gathered}
$$

Here $D(t)$ is the pion propagator, $F_{N}(t)$ is the nucleon form factor related to the vertex $\pi N N$, and $g_{1}^{(\alpha)}(t)$ and $f_{1 a}(t)$ are form factors of the pion block.

The piece of the amplitude for the reaction $p \bar{p}$ (at rest) $\rightarrow \pi^{\circ} \pi^{\circ} \pi^{\circ}, \pi^{\circ} \eta \eta$, which describes the production of two mesons in the $00^{++}$state, is written as follows:

$$
A_{p \bar{p} \rightarrow \text { mesons }}=A_{1}\left(s_{23}\right)+A_{2}\left(s_{13}\right)+A_{3}\left(s_{12}\right)
$$

The amplitude $A_{k}\left(s_{i j}\right)$ corresponds to the process with the "last interaction" of particles $i j$, while the particle $k$ remains a spectator.

The amplitude $A_{1}\left(s_{23}\right)$ for the spectra $\pi \pi$ and $\eta \eta$ has the following form $(b=\pi \pi, \eta \eta)$ :

$$
A_{1}\left(s_{23}\right)=K_{p \bar{p} \pi, a}\left(s_{23}\right)(1-i \rho K)_{a b}^{-1}, K_{p \bar{p} \pi, a}\left(s_{i j}\right)=\left(\sum_{\alpha} \frac{\Lambda_{p \bar{p} \pi}^{(\alpha)} g_{a}^{(\alpha)}}{M_{\alpha}^{2}-s_{i j}}+\phi_{p \bar{p} \pi, a} \frac{1 \mathrm{GeV}^{2}+s_{0}}{s_{i j}+s_{0}}\right) .
$$

In the reaction $p \bar{p}$ (at rest) $\rightarrow \pi^{\circ} \pi^{\circ} \pi^{\circ}$, the amplitude is symmetric with respect to the permutation of pion indices: $A_{1}\left(s_{i j}\right)=A_{2}\left(s_{i j}\right)=A_{3}\left(s_{i j}\right)$. The $\pi^{\circ} \pi^{\circ}$ interaction in the reaction $p \bar{p}($ at rest $) \rightarrow \pi^{\circ} \pi^{\circ} \eta$ is determined as follows (below $b=\pi \pi$ ):

$$
A_{1}\left(s_{23}\right)=K_{p \bar{p} \eta, a}\left(s_{23}\right)(1-i \rho K)_{a b}^{-1}, \quad K_{p \bar{p} \eta, a}\left(s_{i j}\right)=\left(\sum_{\alpha} \frac{\Lambda_{p \bar{p} \eta}^{(\alpha)} g_{a}^{(\alpha)}}{M_{\alpha}^{2}-s_{i j}}+\phi_{p \bar{p} \eta, a} \frac{1 \mathrm{GeV}^{2}+s_{0}}{s_{i j}+s_{0}}\right) .
$$

Parameters $\Lambda_{p \bar{p} \pi}^{\alpha}, \phi_{p \bar{p} \pi}, \Lambda_{p \bar{p} \eta}$ and $\phi_{p \bar{p} \eta}$ can be complex quantities with different phases, that follows from the three-particle interaction; more detailed discussion of the amplitude $p \bar{p} \rightarrow$ three mesons is given in Ref. 50]. 


\subsection{Results of the $K$-matrix fit for the $00^{++}$wave in the region below 1900 $\mathrm{MeV}$}

Simultaneous $K$-matrix fit of the $00^{++}$spectra in the mass region $550-1900 \mathrm{MeV}$ performed in 30] pointed out to the existence of five bare states, $f_{0}^{\text {bare }}$. Only two of them, $f_{0}^{\text {bare }}(720)$ and $f_{0}^{\text {bare }}(1810)$, contain a large $s \bar{s}$-component. This means that only two ${ }^{3} P_{0} q \bar{q}$-nonets can be built in the mass region below $1900 \mathrm{MeV}$.

The following requirements provide the ground for the nonet classification of bare states: (1) Nonet partners are orthogonal in the flavour space, i.e. they must have the mixing angle differences (see Eq. (1.9)) equal to $90^{\circ}: \phi_{1}-\phi_{2}=90^{\circ}$ (for this value the corridor $90^{\circ} \pm 5^{\circ}$ is allowed).

(2) Coupling constants $g^{L}$ and $g^{N L}$ (see Table 2) are approximately equal for nonet partners: $g_{1}^{L} \simeq g_{2}^{L}$ and $g_{1}^{N L} \simeq g_{2}^{N L}$.

The standard quark model requires the equality of coupling constants. However, the $s$-dependent vertex functions and loop diagrams violate this equality because of the presence of mass differences for nonet partners. Moreover, the $K$-matrix coupling constants have an additional $s$-dependent factor $\left(1+B^{\prime}(s)\right)^{-1}$ (see Eq. (2.22)): this factor strongly affects the region of small masses (the region of the basic nonet $1^{3} P_{0}$ ), where the thresholds and left-hand singularities of partial amplitude play more significant role.

The fitting to experimental data (1.4)-(1.7) resulted in two solutions, I and II. First, let us sum up the results for Solution I:

$$
\begin{array}{lll}
\text { Type of the state } & \text { Flavour wave function } \\
f_{0}^{\text {bare }}(720) \rightarrow 1^{3} P_{0} q \bar{q} & 0.40 n \bar{n}-0.92 s \bar{s} \\
f_{0}^{\text {bare }}(1260) \rightarrow 1^{3} P_{0} q \bar{q} & 0.92 n \bar{n}+0.40 s \bar{s} \\
f_{0}^{\text {bare }}(1600) \rightarrow 2^{3} P_{0} q \bar{q} & 0.995 n \bar{n}-0.10 s \bar{s} \\
f_{0}^{\text {bare }}(1810) \rightarrow 2^{3} P_{0} q \bar{q} & 0.10 n \bar{n}+0.995 s \bar{s} \\
f_{0}^{\text {bare }}(1235) \rightarrow \text { Glueball } \rightarrow & 0.91 n \bar{n}+0.42 s \bar{s} .
\end{array}
$$

In Eq. (3.7) the "flavour wave function" is introduced for the glueball: it describes the flavour content of intermediate state for the glueball decay: see Fig. 4c.

Now let us summarize the results for Solution II:

$$
\begin{array}{lll}
\text { Type of the state } & \text { Flavour wave function } \\
f_{0}^{\text {bare }}(720) \rightarrow 1^{3} P_{0} q \bar{q} & 0.40 n \bar{n}-0.92 s \bar{s} \\
f_{0}^{\text {bare }}(1260) \rightarrow 1^{3} P_{0} q \bar{q} & 0.92 n \bar{n}+0.40 s \bar{s} \\
f_{0}^{\text {bare }}(1235) \rightarrow 2^{3} P_{0} q \bar{q} & 0.74 n \bar{n}+0.67 s \bar{s} \\
f_{0}^{\text {bare }}(1810) \rightarrow 2^{3} P_{0} q \bar{q} & 0.67 n \bar{n}-0.74 s \bar{s} \\
f_{0}^{\text {bare }}(1600) \rightarrow \text { Glueball } \rightarrow & 0.91 n \bar{n}+0.42 s \bar{s} .
\end{array}
$$

The quality of data description by Solutions I and II can be seen in Figs. 6-3.4 (dashed and solid curves, respectively).

\subsection{The resonances: are they bumps or dips in the spectra?}

During decades the search for meson resonances meant the search for bumps in the particle spectra. Only recently the understanding came that it is not always so, and the resonance $f_{0}(980)$ provided us with an example. In the peripheric $\pi \pi$-spectra it reveals itself as a dip, and a set of papers was devoted to this phenomenon, see [9, 51]. The study of $00^{++}$wave proved that meson resonances in the region 1000-1600 MeV appear not only as bumps or dips, but as shoulders in the spectra as well. The main particular feature of the resonance is not a bump or a dip in the spectrum, but a circle on the Argand diagram. 
Figures 10 and 11 demonstrate the Argand diagrams relevant to the fits of spectra under discussion. In Fig. 10 one can see the $00^{++}$amplitudes $A_{\pi \pi \rightarrow \pi \pi}, A_{\pi \pi \rightarrow K \bar{K}}, A_{\pi \pi \rightarrow \eta \eta}$ and $A_{\pi \pi \rightarrow \eta \eta^{\prime}}$ as functions of the energy. Indeed, there exist rather legible circles obtained for the resonances $f_{0}(980), f_{0}(1300)$, $f_{0}(1500)$ and $f_{0}(1780)$. The manifestation of resonances $f_{0}(980)$ and $f_{0}(1300)$ in the form of circles is rather clear for the amplitudes $A_{\pi(t) \pi \rightarrow \pi \pi}$ at large $|t|$, see Fig. 11.

\subsection{Resonance $f_{0}(980)$ : is it the $K \bar{K}$-molecule or the descendant of the lightest scalar $q \bar{q}$-states?}

This is a principal problem for the $q \bar{q}$-systematics that was firstly investigated in [14] by using the $K$ matrix analysis for the low-energy part of the $00^{++}$-wave. Following this paper, we provide arguments that $f_{0}(980)$ is the descendant of the lightest $q \bar{q}$-states.

Two poles correspond to the resonance $f_{0}(980)$, with the following complex masses: $M=1015-i 46$ $\mathrm{MeV}$ on the second sheet (under the $\pi \pi$ cut) and $M=936-i 238 \mathrm{MeV}$ on the third sheet (under the $\pi \pi$ and $K \bar{K}$ cuts). The second pole, at $936-i 238 \mathrm{MeV}$, appears because of the well-known doubling of poles affected by the proximity of the $K \bar{K}$-threshold - for example, see Refs. [51, 52]. The first pole at $1015-i 46 \mathrm{MeV}$ dominates in the $\pi \pi$-spectrum, providing a sharp dip in the $\pi \pi \rightarrow \pi \pi$ spectrum or a bump in the spectrum $\pi \pi(t) \rightarrow \pi \pi$ at large $|t|$ [24]. Let us study the dynamics of this pole when the decays into the channels $1=\pi \pi$ and $2=K \bar{K}$ are subsequently switched on and off. To this end, let us make the following replacement in the $K$-matrix amplitude:

$$
g_{1}(720) \rightarrow \xi_{1} g_{1}(720), \quad g_{2}(720) \rightarrow \xi_{2} g_{2}(720),
$$

with the parameters $\xi_{a}$ varying in the interval $0 \leq \xi_{a} \leq 1$. At $\xi_{1} \rightarrow 0$ and $\xi_{2} \rightarrow 0$ the decay channels for the lightest $00^{++}$state are switched off, and we deal with the "bare" state, in terms of Refs. [29, 30]. At $\xi_{1}=\xi_{2}=1$ the real case is restored. At $\xi_{1} \simeq \xi_{2} \simeq 0$ the mass of the bare state is in the vicinity of $720 \mathrm{MeV}$, while, with the increase of $\xi_{a}$, the lightest scalar state acquires the components of real mesons, $\pi \pi$ and $K \bar{K}$, and, due to transitions to these states, it mixes with the other scalar ones. As a result, the mass of the lightest scalar state increases, approaching the region around 1000 $\mathrm{MeV}$. At $\xi_{1}=\xi_{2}=1$ the pole of the amplitude is at

$$
M(\text { real position })=1015-i 46 \mathrm{MeV},
$$

i.e. near the $K \bar{K}$-threshold. Therefore, the $K \bar{K}$-component is of the quasi-molecular type: the relative momentum of $K$-masons is small, hence the mean distance between the $K$-mesons is comparatively large. However, one can see that the $K \bar{K}$-component weakly affects the formation of the final state. Indeed, let us switch off the $K \bar{K}$-state, i.e. let us put in the amplitude $\xi_{1}=1$ and $\xi_{2}=0$. Then, the pole appears in the point:

$$
M(\text { without } K \bar{K})=979-i 53 \mathrm{MeV} \text {. }
$$

The mass shift

$$
M(\text { real position })-M(\text { without } K \bar{K})=36+i 7 \mathrm{MeV}
$$

is actually not large, thus proving the role of the $K \bar{K}$-component in the formation of the real $f_{0}(980)$ state be insignificant.

Concluding, the $K$-matrix analysis of $00^{++}$-wave restores the following picture of the formation of $f_{0}(980)$. Before mixing, there existed the lightest scalar/isoscalar $q \bar{q}$-state $f_{0}^{b a r e}(720 \pm 100)$, with the flavour wave function close to the octet one:

$$
\begin{aligned}
& \psi_{\text {flavour }}(720)=\operatorname{Cos} \theta_{S} \psi_{8}-\operatorname{Sin} \theta_{S} \psi_{1}, \quad \theta_{S}=14^{\circ} \pm 12^{\circ} \\
& \psi_{1}=\frac{1}{\sqrt{3}}(u \bar{u}+d \bar{d}+s \bar{s}) \quad \psi_{8}=\frac{1}{\sqrt{6}}(u \bar{u}+d \bar{d})-\sqrt{\frac{2}{3}} s \bar{s}
\end{aligned}
$$


The mixing with other states, which is going through the transition $f_{0}^{\text {bare }}(720) \rightarrow \pi \pi$, leads to the formation of the resonance with the characteristics which are almost the same as those observed at the experiment, see Eq. (3.11). The onset of the $K \bar{K}$-component $f_{0}^{\text {bare }}(720) \rightarrow K \bar{K}$ affects relatively small shift of mass and width, see Eq. (3.12).

It should be noted that direct measurements also point out that $f_{0}(980)$ has considerable small- $r$ components: the production of $f_{0}(980)$ is not suppressed in the reaction $\pi^{-} p \rightarrow f_{0}(980) p$ at large momentum transfers [24, 53] as well as in radiative $J / \psi$ decay [54].

One should pay attention to the fact that the lightest scalar/isoscalar state, that is, $\eta$-meson, also has flavour wave function close to the octet one: $\eta=\operatorname{Cos} \theta_{P} \psi_{8}-\operatorname{Sin} \theta_{P} \psi_{1}$ with $\theta_{P}=-16.7^{\circ} \pm 2.8^{\circ}$ [12.

\subsection{The wave $I J^{P C}=10^{++}$}

Two isovector/scalar resonances are clearly seen in the reactions (1.4) [18, 19]. The lightest of them is a well-known $a_{0}(980)$, while the next resonance is the recently discovered $a_{0}(1450)$ : according to PDG compilation [9], its mass is $1450 \pm 40 \mathrm{MeV}$ and the width is $\Gamma=270 \pm 40 \mathrm{MeV}$. Note that the fitting to the latest high statistics data [30, 36, 56] provided us with a greater mass value - it is equal to $1520 \pm 40 \mathrm{MeV}$.

For the description of the scalar/isoscalar amplitude, in Ref. [36] the two-pole $4 \times 4$ matrix was used, with the channel notations $1=\pi \eta, 2=K \bar{K}, 3=\pi \eta^{\prime}, 4=$ multiparticle states. The couplings to the two-meson channels are defined by the quark combinatoric relations, see Table 3: we remind that the constants $g^{L}$ are the same for all multiplet members. At the first stage of the fit, the coupling constants of the lightest resonance $a_{0}$ have been varied in the interval limited by the constants $g^{L}\left[f_{0}^{\text {bare }}(720)\right]$ and $g^{L}\left[f_{0}^{\text {bare }}(1260)\right]$. In all the variants of the fit, the coupling $g^{L}\left[a_{0}^{\text {bare }}\right.$ (lightest state) $]$ was found to be rather close to $g^{L}\left[f_{0}^{b a r e}(1260)\right]$ : in the final variant of the fit these coupling constants are taken equal to each other. The couplings of the next isovector/scalar resonance to two mesons are also fixed: they are equal to each other for all the multiplet members $2^{3} P_{0}$.

The fit allowed us to find two solutions for the wave $10^{++}$, which practically coincide for the resonance sector and differ for background terms. Positions of the amplitude poles and relevant bare states are shown in Eqs. (1.14) and (1.15).

In Ref. [41], the hypothesis was discussed about resonances $a_{0}(980)$ and $f_{0}(980)$ belonging to a special class of states (minions), which are loosely bound to hadron channels: the small widths of $a_{0}(980)$ and $f_{0}(980)$ were considered as arguments in favour of that particular nature. The characteristics of $a_{0}(980)$ provide a good opportunity to check this hypothesis, because actually the components of real mesons yielded by the decays $a_{0}(980) \rightarrow \pi \eta, K \bar{K}$ do not influence this state, see Eqs. (1.14) and (1.15). However, the $K$-matrix data fittings [36] proved that $g^{L}\left[f_{0}^{b a r e}(964)\right]$ is not small, being of the order of a standard hadronic value, for the small width of $a_{0}(980)$ is related not to the probability of the decay, as it could follow from the minion nature of $a_{0}(980)$, but to the threshold effect. It should be emphasized that this result is also seen in the $T$-matrix analysis of the data [19, 56]. 
Table 1

Coupling constants of the glueball decaying to two pseudoscalar mesons, in the leading and next-to leading terms of $1 / N$ expansion. $\Theta$ is the mixing angle for $\eta-\eta^{\prime}$ mesons: $\eta=n \bar{n} \cos \Theta-s \bar{s} \sin \Theta$ and $\eta^{\prime}=n \bar{n} \sin \Theta+s \bar{s} \cos \Theta$.

\begin{tabular}{|c|c|c|c|}
\hline Channel & $\begin{array}{c}\text { Glueball decay } \\
\text { couplings in the } \\
\text { leading term of } \\
1 / N \text { expansion. }\end{array}$ & $\begin{array}{c}\text { Glueball decay } \\
\text { couplings in the } \\
\text { next-to-leading term } \\
\text { of } 1 / N \text { expansion. }\end{array}$ & $\begin{array}{c}\text { Iden- } \\
\text { tity } \\
\text { factor }\end{array}$ \\
\hline$\pi^{0} \pi^{0}$ & $G^{L}$ & 0 & $1 / 2$ \\
$\pi^{+} \pi^{-}$ & $G^{L}$ & 0 & 1 \\
$K^{+} K^{-}$ & $\sqrt{\lambda} G^{L}$ & 0 & 1 \\
$K^{0} K^{0}$ & $\sqrt{\lambda} G^{L}$ & 0 & 1 \\
$\eta \eta$ & $G^{L}\left(\cos ^{2} \Theta+\lambda \sin ^{2} \Theta\right)$ & $2 G^{N L}\left(\cos \Theta-\sqrt{\frac{\lambda}{2}} \sin \Theta\right)^{2}$ & $1 / 2$ \\
$\eta^{\prime}$ & $G^{L}(1-\lambda) \sin \Theta \cos \Theta$ & $2 G^{N L}\left(\cos \Theta-\sqrt{\frac{\lambda}{2}} \sin \Theta\right) \times$ & 1 \\
$\eta^{\prime} \eta^{\prime}$ & $G^{L}\left(\sin ^{2} \Theta+\lambda \cos ^{2} \Theta\right)$ & $2 G^{N L}\left(\sin \Theta+\sqrt{\frac{\lambda}{2}} \cos \Theta\right)^{2}$ & $1 / 2$ \\
\hline
\end{tabular}


Table 2

Coupling constants of $q \bar{q}$-meson decaying to two pseudoscalar mesons in the leading and next-to-leading terms of the $1 / N$ expansion. $\phi$ is the mixing angle for $n \bar{n}$ and $s \bar{s}$ states, see (1.9).

\begin{tabular}{|c|c|c|}
\hline Channel & $\begin{array}{c}\text { Decay couplings of } \\
q \bar{q} \text { mesons } \\
\text { in leading term } \\
\text { of } 1 / N \text { expansion. }\end{array}$ & $\begin{array}{l}\text { Decay couplings of } \\
\qquad q \bar{q} \text { mesons } \\
\text { in next-to-leading term } \\
\text { of } 1 / N \text { expansion. }\end{array}$ \\
\hline$\pi^{0} \pi^{0}$ & $g^{L} \cos \phi / \sqrt{2}$ & 0 \\
\hline$\pi^{+} \pi^{-}$ & $g^{L} \cos \phi / \sqrt{2}$ & 0 \\
\hline$K^{+} K^{-}$ & $g^{L}(\sqrt{2} \sin \phi+\sqrt{\lambda} \cos \phi) / \sqrt{8}$ & 0 \\
\hline$K^{0} K^{0}$ & $g^{L}(\sqrt{2} \sin \phi+\sqrt{\lambda} \cos \phi) / \sqrt{8}$ & 0 \\
\hline$\eta \eta$ & $\begin{array}{l}g^{L}\left(\cos ^{2} \Theta \cos \phi / \sqrt{2}+\right. \\
\left.\sqrt{\lambda} \sin \phi \sin ^{2} \Theta\right)\end{array}$ & $\begin{array}{r}\sqrt{2} g^{N L}\left(\cos \Theta-\sqrt{\frac{\lambda}{2}} \sin \Theta\right) \times \\
(\cos \phi \cos \Theta-\sin \phi \sin \Theta)\end{array}$ \\
\hline$\eta \eta^{\prime}$ & $\begin{array}{r}g^{L} \sin \Theta \cos \Theta(\cos \Phi / \sqrt{2}- \\
\sqrt{\lambda} \sin \phi)\end{array}$ & $\begin{array}{c}\sqrt{\frac{1}{2}} g^{N L}\left[\left(\cos \Theta-\sqrt{\frac{\lambda}{2}} \sin \Theta\right) \times\right. \\
(\cos \phi \sin \Theta+\sin \phi \cos \Theta) \\
+\left(\sin \Theta+\sqrt{\frac{\lambda}{2}} \cos \Theta\right) \times \\
(\cos \phi \sin \Theta-\sin \phi \cos \Theta)]\end{array}$ \\
\hline$\eta^{\prime} \eta^{\prime}$ & $\begin{array}{l}g^{L}\left(\sin ^{2} \Theta \cos \phi / \sqrt{2}+\right. \\
\left.\sqrt{\lambda} \sin \Phi \cos ^{2} \Theta\right)\end{array}$ & $\begin{array}{r}\sqrt{2} g^{N L}\left(\sin \Theta+\sqrt{\frac{\lambda}{2}} \cos \Theta\right) \times \\
(\cos \phi \cos \Theta+\sin \phi \sin \Theta)\end{array}$ \\
\hline
\end{tabular}



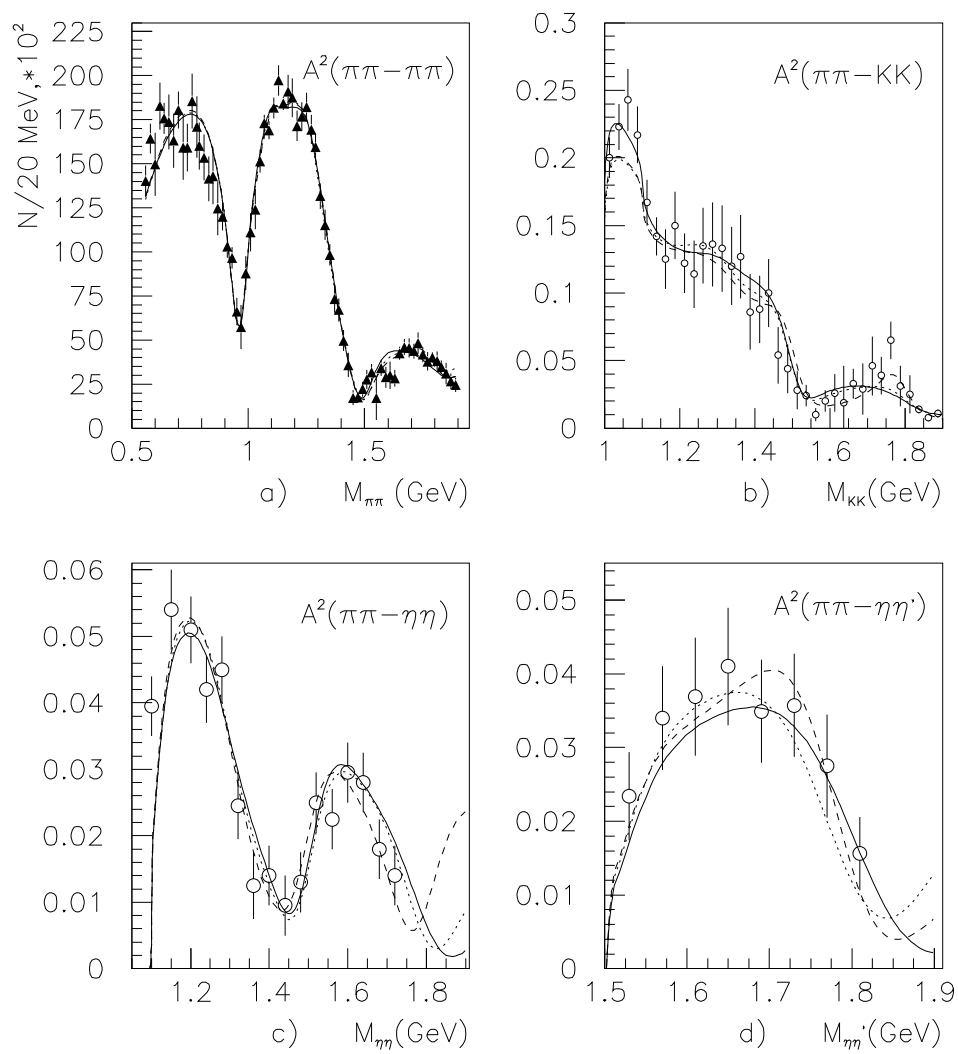

Fig. 6. $S$ wave amplitudes squared and their description in Ref. [30]: solid curve stands for Solution 

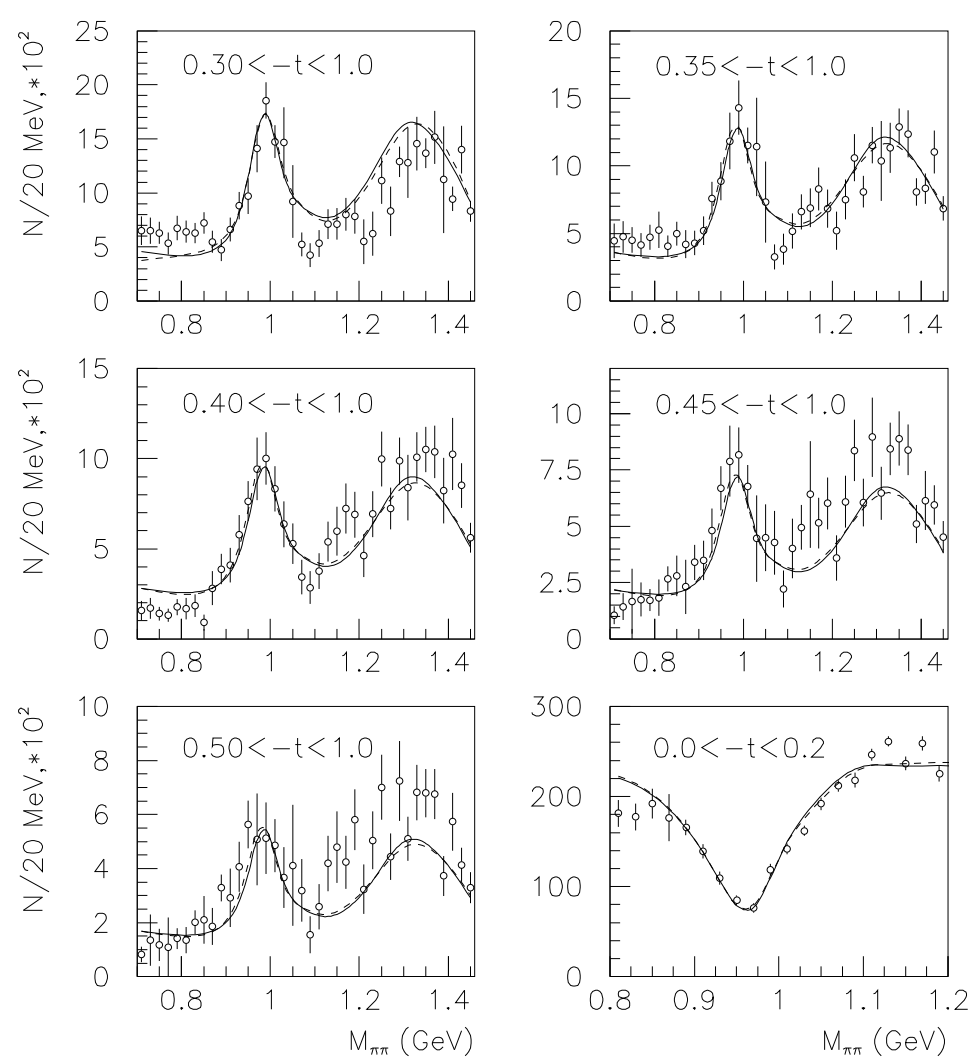

II.

Fig. 7. Event numbers versus invariant mass of the $\pi \pi$-system selected for various intervals of the momentum transfer squared $t$. Solid curve stands for Solution II, dashed one to Solution I. 

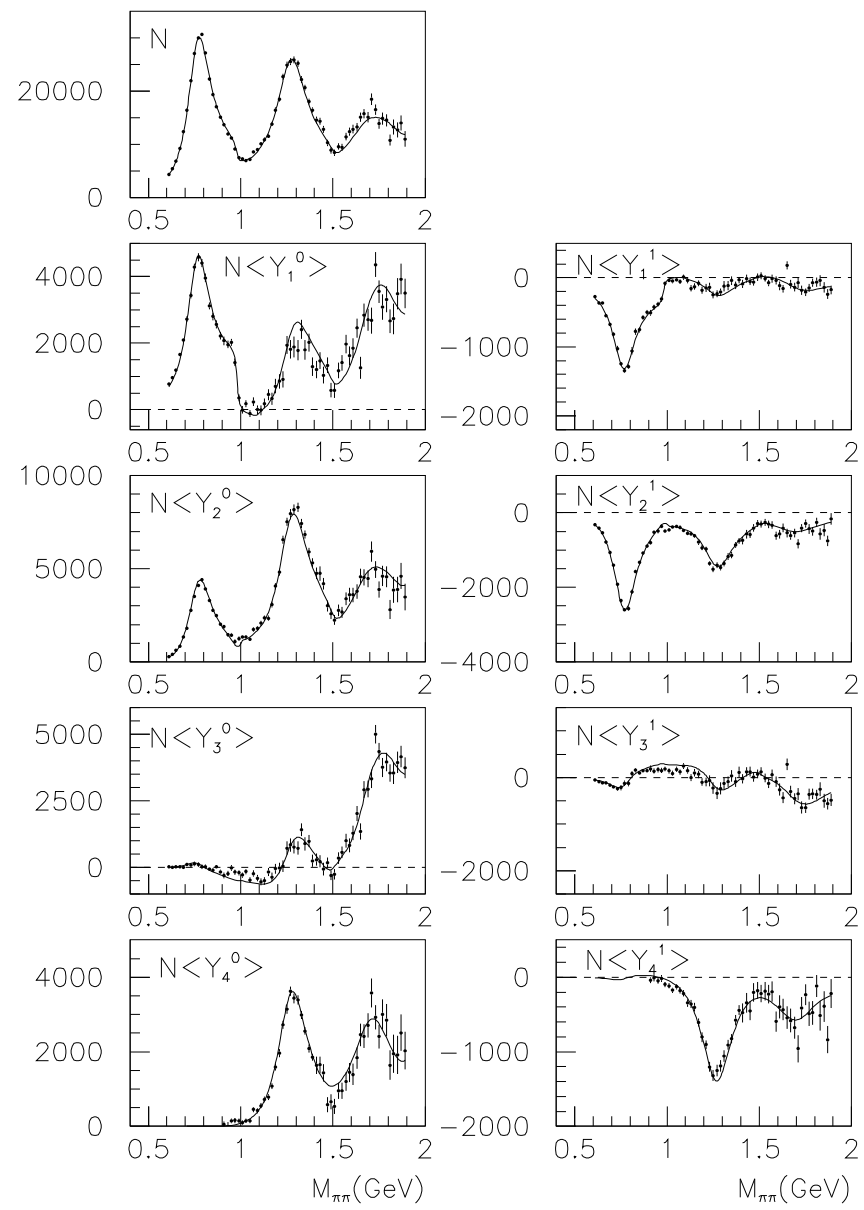

Fig. 8. Description of the angle moments for the $\pi \pi$ distributions measured in the reaction $\pi^{-} p \rightarrow n \pi^{-} \pi^{+}$[27]; Solution II [30]. 

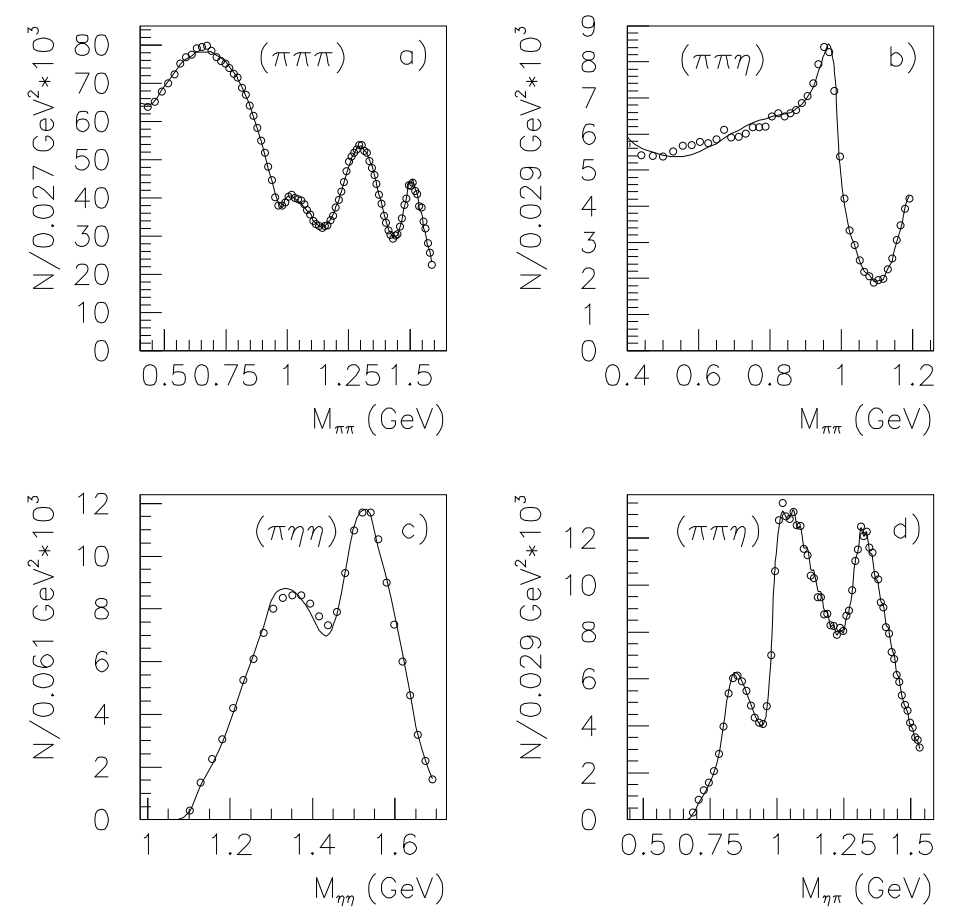

Fig. 9. The $\pi^{0} \pi^{0}$ spectra in the reactions $p \bar{p} \rightarrow \pi^{0} \pi^{0} \pi^{0}, p \bar{p} \rightarrow \eta \pi^{0} \pi^{0}$ and $\eta \eta$ spectrum in the reaction $p \bar{p} \rightarrow \pi^{0} \eta \eta$ and $\eta \pi^{0}$ spectrum in the reaction $p \bar{p} \rightarrow \pi^{0} \pi^{0} \eta$. Curves correspond to Solution II [30. 

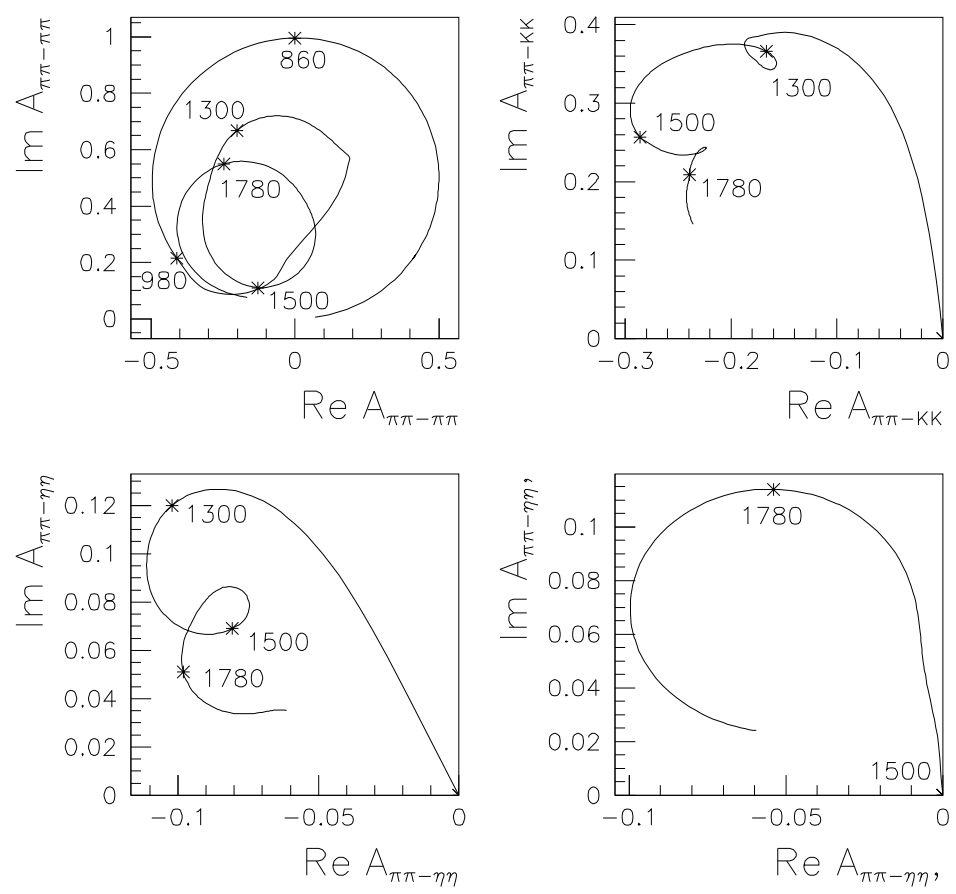

Fig. 10. Argand diagram for the unitary S-wave amplitudes in the reactions $\pi \pi \rightarrow \pi \pi, \pi \pi \rightarrow K \bar{K}$, $\pi \pi \rightarrow \eta \eta$ and $\pi \pi \rightarrow \eta \eta^{\prime}$ [30]. 

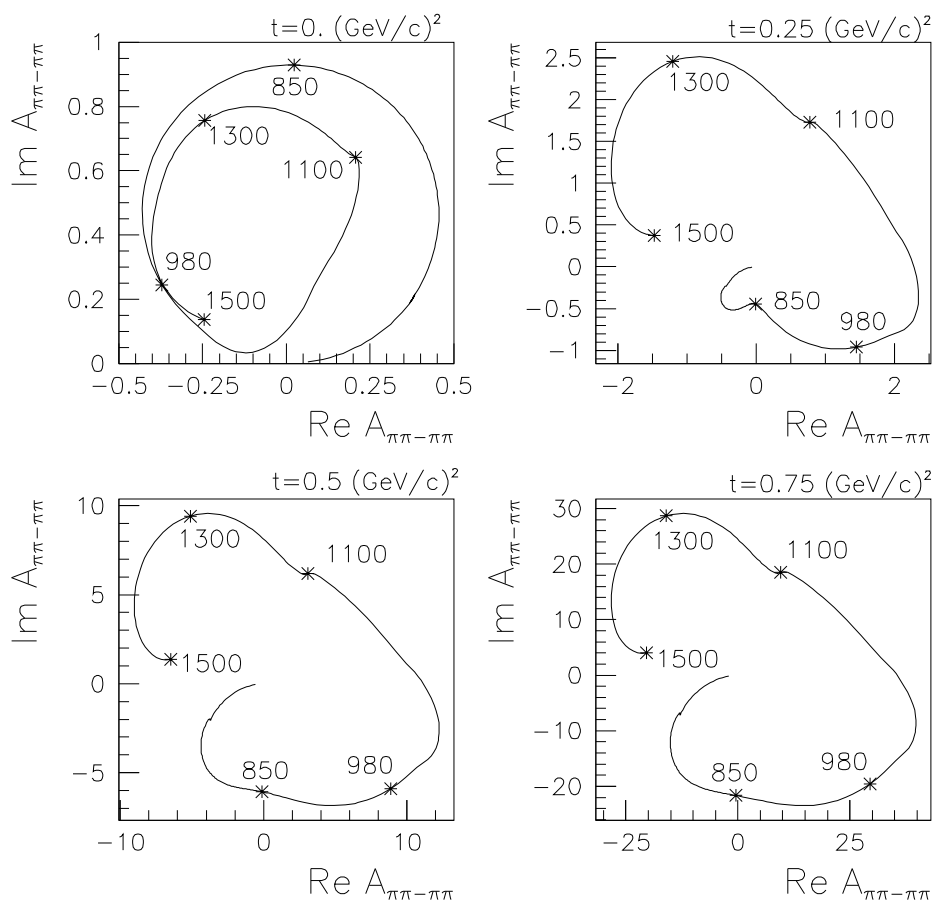

Fig. 11. Argand diagram for the isoscalar S-wave $\pi \pi(t) \rightarrow \pi \pi$ scattering amplitude 30$]$ at different momentum transfers squared, $t$.

\section{6 $K$-matrix analysis for the $K \pi S$-wave}

Partial-wave analysis of the $K^{-} \pi^{+}$system for the reaction $K^{-} p \rightarrow K^{-} \pi^{+} n$ at $11 \mathrm{GeV} / \mathrm{c}$ was carried out in Ref. [37], where two alternative solutions (A and B), which differ only in the region above 1800 $\mathrm{MeV}$, were found for the $S$-wave. In that paper the $T$-matrix fit for the $K \pi S$-wave was performed as well, though independently for the regions $850-1600 \mathrm{MeV}$ and $1800-2100 \mathrm{MeV}$. In the first region, the resonance $K_{0}^{*}(1430)$ was found:

$$
M_{R}=1429 \pm 9 \mathrm{MeV}, \quad \Gamma=287 \pm 31 \mathrm{MeV} .
$$

In the second mass region, Solutions A and B provided the following parameters for the description of the resonance $K_{0}^{*}(1950)$ :

$$
\begin{array}{ll}
\text { Solution } A & M_{R}=1934 \pm 28 \mathrm{MeV}, \quad \Gamma=174 \pm 98 \mathrm{MeV}, \\
\text { Solution } B & M_{R}=1955 \pm 18 \mathrm{MeV}, \quad \Gamma=228 \pm 56 \mathrm{MeV} .
\end{array}
$$

The necessity to improve this analysis is obvious. First, the mass region $1600-1800 \mathrm{MeV}$, where the amplitude varies quickly, must be included into consideration. As was stressed above, it is wellknown that, due to the strong interference, the resonance reveals itself not only as a bump in the spectrum but also as a dip or a shoulder; likewise, the resonances appear in the $00^{++}$wave. Second, the interference effects are the source of ambiguities. It is worth noting that ambiguities in $00^{++}$wave were successfully eliminated in the analyses of Refs. [30, 36], that was due to the simultaneous fit of 
different meson spectra only. For the wave $\frac{1}{2} 0^{+}$, the available data are not copious, hence one may suspect that the solution found in Ref. [37] is not unique.

The $K$-matrix re-analysis of the $K \pi S$-wave has been carried out in Ref. 42] with a purpose:

(i) to restore the masses and couling constants of the bare states for the wave $\frac{1}{2} 0^{+}$, in order to establish the $q \bar{q}$-classification;

(ii) to find out all possible $K$-matrix solutions for the $K \pi S$-wave in the mass region up to 2000 $\mathrm{MeV}$.

The $S$-wave $K \pi$ scattering amplitude extracted from the reaction $K^{-} p \rightarrow K^{-} \pi^{+} n$ at small momentum transfers is a sum of two components, with isotopic spins $\frac{1}{2}$ and $\frac{3}{2}$ :

$$
A_{S}=A_{S}^{1 / 2}+\frac{1}{2} A_{S}^{3 / 2}=\left|A_{S}\right| e^{i \phi_{S}}
$$

where $\left|A_{S}\right|$ and $\phi_{S}$ are measurable quantities entering the $S$-wave amplitude [37]. The part of $S$ wave amplitude with the isotopic spin $I=3 / 2$ has a non-resonance behaviour at the energies under consideration, so it can be parametrised as follows:

$$
A_{S}^{3 / 2}(s)=\frac{\rho_{K \pi}(s) a_{3 / 2}(s)}{1-i \rho_{K \pi}(s) a_{3 / 2}(s)},
$$

where $a_{3 / 2}(s)$ is a smooth function and $\rho_{K \pi}(s)$ is the $K \pi$ phase space factor.

For the description of the $A_{S}^{1 / 2}$ amplitude, in 42 the $3 \times 3 \mathrm{~K}$-matrix was used, with the following channel notations: $1=K \pi, 2=K \eta^{\prime}, 3=K \pi \pi \pi+$ multimeson states. The account of the channel $K \eta$ does not influence the data description, since the transition $K \pi \rightarrow K \eta$ is suppressed [37, that also agrees with quark combinatoric results, see Table 3. In Ref. 42 the fitting to the wave $\frac{1}{2} 0^{+}$ was performed with the parametrization of $K_{a b}$ given in (3.1). The analysed data for the reaction $K^{-} p \rightarrow K^{-} \pi^{+} n$ were extracted with small momentum transfers $\left(|t|<0.2 \mathrm{GeV}^{2}\right)$, and at the first stage the data were fitted to the unitary amplitude (3.1). At the next stage, the $t$-dependence was introduced into the $K$-matrix amplitude. The amplitude $K \pi(t) \rightarrow K \pi(\pi(t)$ stands for virtual pion) is equal to $A_{S}^{1 / 2}=K_{1 a}(t)(I-i \rho K)_{a 1}^{-1}$ : the parametrization of the matrix $K_{1 a}(t)$ is written in (3.3).

Coupling constants are determined by the rules of quark combinatorics, they are presented in Table 3. In Ref. [42] only the leading terms in the $1 / N$ expansion were taken into consideration: in this case, coupling constants were fixed by the fit of the $00^{++}$and $10^{++}$waves, because $g^{L}$ is a common parameter for all the nonet members.

The description of the $\frac{1}{2} 0^{+}$-wave has been performed under two assumptions, namely, with twoand three-pole structure of the wave in the mass region below $2000 \mathrm{MeV}$.

In Ref. [37] two solutions, $\mathrm{A}$ and B, were found for the wave $\frac{1}{2} 0^{+}$; they differ at $M_{\pi K}>1800 \mathrm{MeV}$ only. Correspondingly, in Ref. [42] the two two-pole $K$-matrix solutions, (A-1) and (B-1), have been obtained. The positions of amplitude poles are practically the same for both solutions; they are shown in Eq. (1.16). The description of data is shown in Fig. 12. The mass of the first resonance, see (1.16), does not differ strongly from that obtained in [37], see (3.15), but the width of the resonance found in the $K$-matrix solution is twice as less. This follows from the doubling of poles in the $K$-matrix solution due to the correct account of the $K \eta^{\prime}$ threshold. The mass of the second resonance decreased for the $K$-matrix solution, as compared to the result of Ref. [37], in more than $100 \mathrm{MeV}$.

The masses of bare kaon states related to the two-pole solution are given in Eq. (1.17). The mass of lightest state is $1200_{-110}^{+60} \mathrm{MeV}$, i.e. this scalar kaon is located in the same mass region as the other scalars, candidates for basic $1^{3} P_{0}$ nonet members.

The description of data in the three-pole $K$-matrix fit is shown in Fig. 13. The region of high masses, $M_{K \pi}>1700 \mathrm{MeV}$ is described by two poles in Solutions (A-2) and (B-2). However, the two-pole structure of the amplitude at $M_{K \pi}>1700 \mathrm{MeV}$ did not influence the characteristics of two 
low-located resonances - they are identical in Solutions (A-2) and (B-2). In Solution (B-3) the region $M_{K \pi}<1600 \mathrm{MeV}$ is described by two poles. Positions of bare states in Solution (B-3) are given in (1.18), the corresponding positions of poles are given in Eq. (1.19).

Solid curves in Figs. 12 and 13 represent the description of the $K \pi$ wave by the unitary amplitude, and dashed lines represent the fits, where the $t$-dependence of the $K \pi$ amplitude is taken into account. It is seen that the $t$-dependence allows us to get better description of phase shifts around $1700 \mathrm{MeV}$. It should be noted that in this region as well as in the mass region above $2000 \mathrm{MeV}$ for Solution A, the data under consideration violate the unitary limit. It is hardly possible that rather strong violation of unitarity is a consequence of the amplitude $t$-dependence; it is more likely related to the underestimation of systematic errors in the partial-wave analysis of Ref. [37] in those regions. The inclusion of the $t$-dependence into fitting procedure does not affect strongly the masses of bare states and positions of pole of the amplitude. As a rule, the masses of bare states found in the $t$-dependent fit are less in 20-30 MeV than masses obtained in the $t$-independent fits. 
Table 3

Coupling constants for the transitions $K_{0}^{0} \rightarrow$ two mesons and $a_{0}^{-} \rightarrow$ two mesons in the leading and next-to-leading terms of the $1 / N$ expansion.

\begin{tabular}{|l|c|c|}
\hline Channel & $\begin{array}{c}\text { Couplings for } \\
\text { leading terms }\end{array}$ & $\begin{array}{c}\text { Couplings for } \\
\text { next-to-leading terms }\end{array}$ \\
\hline$K^{+} \pi^{-}$ & $g^{L} / 2$ & 0 \\
$K^{0} \pi^{0}$ & $-g^{L} / \sqrt{8}$ & 0 \\
$K^{0} \eta$ & $(\cos \Theta / \sqrt{2}-\sqrt{\lambda} \sin \Theta) g^{L} / 2$ & $(\sqrt{2} \cos \Theta-\sqrt{\lambda} \sin \Theta) g^{N L} / 2$ \\
$K^{0} \eta^{\prime}$ & $(\sin \Theta / \sqrt{2}+\sqrt{\lambda} \cos \Theta) g^{L} / 2$ & $(\sqrt{2} \sin \Theta-\sqrt{\lambda} \cos \Theta) g^{N L} / 2$ \\
\hline$K^{-} K^{0}$ & $g^{L} \sqrt{\lambda} / 2$ & 0 \\
$\pi^{-} \eta$ & $g^{L} \cos \Theta / \sqrt{2}$ & $(\sqrt{2} \cos \Theta-\sqrt{\lambda} \sin \Theta) g^{N L} / 2$ \\
$\pi^{-} \eta^{\prime}$ & $g^{L} \sin \Theta / \sqrt{2}$ & $(\sqrt{2} \sin \Theta-\sqrt{\lambda} \cos \Theta) g^{N L} / 2$ \\
\hline
\end{tabular}



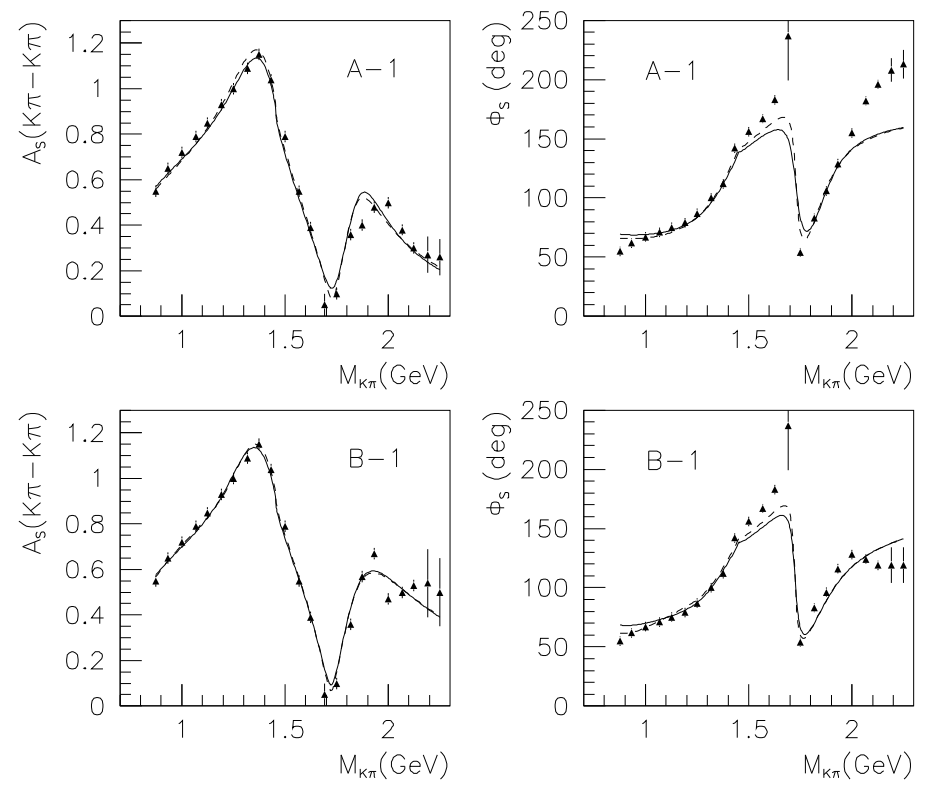

Fig. 12. Description of data in Ref. [37] in the two-pole $K$-matrix fit: Solutions (A-1) and (B-1). Solid curves correspond to the solution found for the unitary amplitude, dashed line stands for the fit with the $t$-dependent $K$-matrix. 

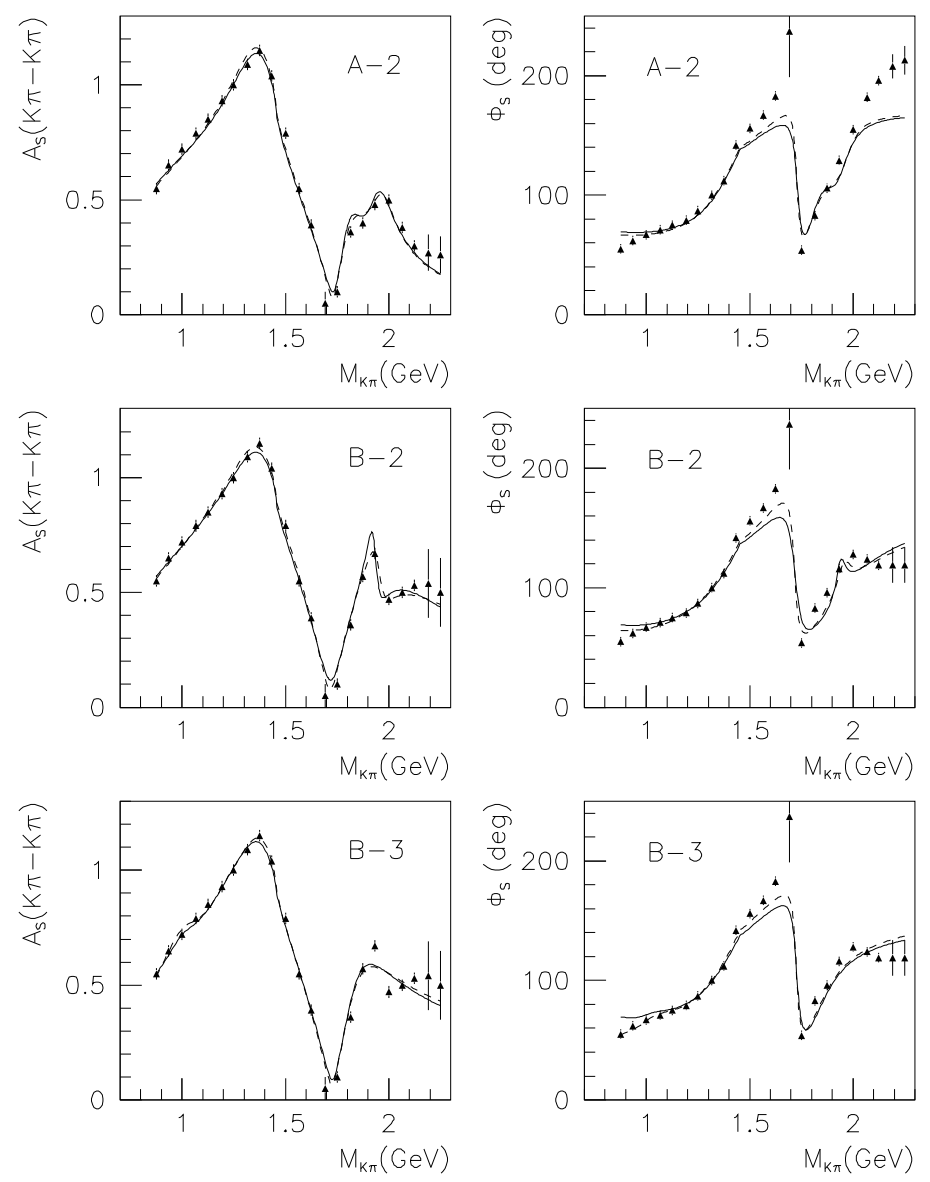

Fig. 13. Description of data in Ref. [37 in the three-pole $K$-matrix fit: Solutions (A-2), (B-2) and (B-3).

\section{Propagator matrix: analysis of the $\left(I J^{P C}=00^{++}\right)$-wave}

Here we are summing up the results of the analysis of the $00^{++} S$-wave performed in [6, 7], in terms of propagator matrix ( $D$-matrix). The $D$-matrix technique is based on the dispersion relation $N / D$-method, it allows us to reconstruct the amplitude, which is analytical on the whole complex $s$ plane. We discuss the effects which are due to the resonance overlapping and mixing: mass shifts and accumulation of widths by one of neighbouring resonances. The expansion of the physically observed states in a series with respect to initial (non-mixed) ones is performed.

The investigation is done for the $00^{++}$wave, though the method can be easily generalised for other waves using the technique developped in Refs. 44, 45. 


\subsection{The mixing of two unstable states}

In the case of two resonances, the propagator of the state 1 is determined by the diagrams of Fig. 14a. With all these processes taken into account, the propagator of the state 1 is equal to:

$$
D_{11}(s)=\left(m_{1}^{2}-s-B_{11}(s)-\frac{B_{12}(s) B_{21}(s)}{m_{2}^{2}-s-B_{22}(s)}\right)^{-1} .
$$

Here $m_{1}$ and $m_{2}$ are masses of the input states 1 and 2, and the loop diagrams $B_{i j}(s)$ are defined by Eq. (2.21), with the replacement $g^{2}(s) \rightarrow g_{i}(s) g_{j}(s)$. It is helpful to introduce the propagator matrix $D_{i j}$, where the nondiagonal elements $D_{12}=D_{21}$ correspond to the transitions $1 \rightarrow 2$ and $2 \rightarrow 1$ (see Fig. 14b). The matrix reads:

$$
\hat{D}=\left|\begin{array}{cc}
D_{11} & D_{12} \\
D_{21} & D_{22}
\end{array}\right|=\frac{1}{\left(M_{1}^{2}-s\right)\left(M_{2}^{2}-s\right)-B_{12} B_{21}}\left|\begin{array}{cc}
M_{2}^{2}-s, & B_{12} \\
B_{21}, & M_{1}^{2}-s
\end{array}\right| .
$$

Here the following notation is used:

$$
M_{i}^{2}=m_{i}^{2}-B_{i i}(s) \quad i=1,2 .
$$

Zeros of the denominator of the propagator matrix (4.2) define the complex resonance masses after the mixing:

$$
\Pi(s)=\left(M_{1}^{2}-s\right)\left(M_{2}^{2}-s\right)-B_{12} B_{21}=0 .
$$

Let us denote the complex masses of mixed states by $M_{A}$ and $M_{B}$.

Consider a simple model, where the $s$-dependence of the function $B_{i j}(s)$ near the points $s \sim M_{A}^{2}$ and $s \sim M_{B}^{2}$ is assumed to be negligible. Let $M_{i}^{2}$ and $B_{12}$ be constants, then one has:

$$
M_{A, B}^{2}=\frac{1}{2}\left(M_{1}^{2}+M_{2}^{2}\right) \pm \sqrt{\frac{1}{4}\left(M_{1}^{2}-M_{2}^{2}\right)^{2}+B_{12} B_{21}} .
$$

In the case, when the widths of initial resonances 1 and 2 are small (hence the imaginary part of the transition diagram $B_{12}$ is also small), the equation (4.5) turns into the standard formula of quantum mechanics for the split of mixing levels, which become repulsive as a result of the mixing. Then

$$
\hat{D}=\left|\begin{array}{cc}
\frac{\cos ^{2} \theta}{M_{A}^{2}-s}+\frac{\sin ^{2} \theta}{M_{B}^{2}-s} & \frac{-\cos \theta \sin \theta}{M_{A}^{2}-s}+\frac{\sin \theta \cos \theta}{M_{B}^{2}-s} \\
\frac{-\cos \theta \sin \theta}{M_{A}^{2}-s}+\frac{\sin \theta \cos \theta}{M_{B}^{2}-s} & \frac{\sin ^{2} \theta}{M_{A}^{2}-s}+\frac{\cos ^{2} \theta}{M_{B}^{2}-s}
\end{array}\right|, \cos ^{2} \theta=\frac{1}{2}+\frac{1}{2} \frac{\frac{1}{2}\left(M_{1}^{2}-M_{2}^{2}\right)}{\sqrt{\frac{1}{4}\left(M_{1}^{2}-M_{2}^{2}\right)^{2}+B_{12} B_{21}}} .
$$

The states $\mid A>$ and $\mid B>$ are superpositions of initial levels, $\mid 1>$ and $\mid 2>$, as follows:

$$
|A>=\cos \theta| 1>-\sin \theta|2>, \quad| B>=\sin \theta|1>+\cos \theta| 2>.
$$

In general, representation of states $\mid A>$ and $\mid B>$ as superpositions of initial states is valid, when one cannot neglect the $s$-dependence of functions $B_{i j}(s)$ and their imaginary parts are not small. Consider the propagator matrix near $s=M_{A}^{2}$ :

$$
\hat{D}=\frac{1}{\Pi(s)}\left|\begin{array}{cc}
M_{2}^{2}(s)-s & B_{12}(s) \\
B_{21}(s) & M_{1}^{2}(s)-s
\end{array}\right| \simeq \frac{-1}{\Pi^{\prime}\left(M_{A}^{2}\right)\left(M_{A}^{2}-s\right)}\left|\begin{array}{cc}
M_{2}^{2}\left(M_{A}^{2}\right)-M_{A}^{2} & B_{12}\left(M_{A}^{2}\right) \\
B_{21}\left(M_{A}^{2}\right) & M_{1}^{2}\left(M_{A}^{2}\right)-M_{A}^{2}
\end{array}\right| .
$$

In the left-hand side of Eq. (4.8), the singular (pole) terms are the only surviving. The matrix determinant in the right-hand side of (4.8) is equal to zero:

$$
\left[M_{2}^{2}\left(M_{A}^{2}\right)-M_{A}^{2}\right]\left[M_{1}^{2}\left(M_{A}^{2}\right)-M_{A}^{2}\right]-B_{12}\left(M_{A}^{2}\right) B_{21}\left(M_{A}^{2}\right)=0,
$$


This equality follows from Eq. (4.4), which fixes $\Pi\left(M_{A}^{2}\right)=0$. It allows us to introduce the complex mixing angle:

$$
\left|A>=\cos \theta_{A}\right| 1>-\sin \theta_{A} \mid 2>.
$$

The right-hand side of Eq. (4.8) can be rewritten with the use of the mixing angle $\theta_{A}$, as follows:

$$
[\hat{D}]_{s \sim M_{A}^{2}}=\frac{N_{A}}{M_{A}^{2}-s}\left|\begin{array}{cc}
\cos ^{2} \theta_{A} & -\cos \theta_{A} \sin \theta_{A} \\
-\sin \theta_{A} \cos \theta_{A} & \sin ^{2} \theta_{A}
\end{array}\right|
$$

where

$$
N_{A}=\frac{1}{\Pi^{\prime}\left(M_{A}^{2}\right)}\left[2 M_{A}^{2}-M_{1}^{2}-M_{2}^{2}\right], \cos ^{2} \theta_{A}=\frac{M_{A}^{2}-M_{2}^{2}}{2 M_{A}^{2}-M_{1}^{2}-M_{2}^{2}}, \sin ^{2} \theta_{A}=\frac{M_{A}^{2}-M_{1}^{2}}{2 M_{A}^{2}-M_{1}^{2}-M_{2}^{2}} .
$$

We remind that in the formula (4.12) the functions $M_{1}^{2}(s), M_{2}^{2}(s)$ and $B_{12}(s)$ are fixed in the point $s=M_{A}^{2}$. In the case under consideration, when the angle $\theta_{A}$ is a complex quantity, the values $\cos ^{2} \theta_{A}$ and $\sin ^{2} \theta_{A}$ do not determine the probability of states $\mid 1>$ and $\mid 2>$ in $\mid A>$; indeed, the values $\sqrt{N_{A}} \cos \theta_{A}$ and $-\sqrt{N_{A}} \sin \theta_{A}$ are the transition amplitudes $|A>\rightarrow| 1>$ and $|A>\rightarrow| 2>$. Therefore, the corresponding probabilities are equal to $\left|\cos \theta_{A}\right|^{2}$ and $\left|\sin \theta_{A}\right|^{2}$.

In order to analyse the content of the state $|B\rangle$, an analogous expansion of the propagator matrix should be done near the point $s=M_{B}^{2}$. After introducing

$$
\left|B>=\sin \theta_{B}\right| 1>+\cos \theta_{B} \mid 2>,
$$

we have the following expression for $\hat{D}$ in the vicinity of the second pole $s=M_{B}^{2}$ :

$$
[\hat{D}]_{s \sim M_{B}^{2}}=\frac{N_{B}}{M_{B}^{2}-s}\left|\begin{array}{ll}
\sin ^{2} \theta_{B} & \cos \theta_{B} \sin \theta_{B} \\
\sin \theta_{B} \cos \theta_{B} & \cos ^{2} \theta_{B}
\end{array}\right|,
$$

where

$$
N_{B}=\frac{1}{\Pi^{\prime}\left(M_{B}^{2}\right)}\left[2 M_{B}^{2}-M_{1}^{2}-M_{2}^{2}\right], \cos ^{2} \theta_{B}=\frac{M_{B}^{2}-M_{1}^{2}}{2 M_{B}^{2}-M_{1}^{2}-M_{2}^{2}}, \sin ^{2} \theta_{B}=\frac{M_{B}^{2}-M_{2}^{2}}{2 M_{B}^{2}-M_{1}^{2}-M_{2}^{2}} .
$$

In Eq. (4.15) the functions $M_{1}^{2}(s), M_{2}^{2}(s)$ and $B_{12}(s)$ are fixed in the point $s=M_{B}^{2}$.

If $B_{12}$ depends weakly on $s$ and one can neglect this dependence, the angles $\theta_{A}$ and $\theta_{B}$ coincide. But in general they are different. So the formulae for the propagator matrix differ from the standard approach of quantum mechanics by this very point.

Another distinction is related to the type of the level shift afforded by mixing, namely, in quantum mechanics the levels "repulse" each other from the mean value $1 / 2\left(E_{1}+E_{2}\right)$ (see also Eq. (4.5)). Generally, the equation (4.4) can cause both the "repulsion" of masses squared from the mean value, $1 / 2\left(M_{1}^{2}+M_{2}^{2}\right)$, and the "attraction".

The scattering amplitude in the one-channel case is defined by the following expression:

$$
A(s)=g_{i}(s) D_{i j}(s) g_{j}(s) .
$$

In the multichannel case, $B_{i j}(s)$ is a sum of loop diagrams:

$$
B_{i j}(s)=\sum_{n} B_{i j}^{(n)}(s),
$$

$B_{i j}^{(n)}$ is a loop diagram in the channel $n$ with vertex functions $g_{i}^{(n)}, g_{j}^{(n)}$ and phase space factor $\rho_{n}$. Partial scattering amplitude in the channel $n$ is equal to:

$$
A_{n}(s)=g_{i}^{(n)}(s) D_{i j}(s) g_{j}^{(n)}(s) .
$$




\subsection{The overlapping of a large number of resonances: construction of prop- agator matrix}

Consider the construction of the propagator matrix $\hat{D}$ for arbitrary number of resonances. The matrix elements, $D_{i j}$, describe the transition from the input state $i$ (with the bare propagator $\left(m_{i}^{2}-s\right)^{-1}$ ) to the state $j$. They obey the system of linear equations as follows:

$$
D_{i j}=D_{i k} B_{k j}(s)\left(m_{j}^{2}-s\right)^{-1}+\delta_{i j}\left(m_{j}^{2}-s\right)^{-1},
$$

where $B_{i j}(s)$ is the loop diagram for the transition $i \rightarrow j$ and $\delta_{i j}$ is the Kronecker symbol. Let us introduce the diagonal propagator matrix $\hat{d}$ for input states :

$$
\hat{d}=\operatorname{diag}\left(\left(m_{1}^{2}-s\right)^{-1},\left(m_{2}^{2}-s\right)^{-1},\left(m_{3}^{2}-s\right)^{-1} \cdots\right) .
$$

Then the system of linear equations (4.19) can be rewritten in the matrix form as follows:

$$
\hat{D}=\hat{D} \hat{B} \hat{d}+\hat{d} .
$$

One obtains:

$$
\hat{D}=\frac{I}{\left(\hat{d}^{-1}-\hat{B}\right)} .
$$

The matrix $\hat{d}^{-1}$ is diagonal, hence $\hat{D}^{-1}=\left(\hat{d}^{-1}-\hat{B}\right)$ is of the form:

$$
\hat{D}^{-1}=\left|\begin{array}{cccc}
M_{1}^{2}-s & -B_{12}(s) & -B_{13}(s) & \ldots \\
-B_{21}(s) & M_{2}^{2}-s & -B_{23}(s) & \ldots \\
-B_{31}(s) & -B_{32}(s) & M_{3}^{2}-s & \ldots \\
\vdots & \vdots & \vdots & \vdots
\end{array}\right|,
$$

where $M_{i}^{2}$ is defined by Eq. (4.3). Inversing this matrix, we obtain a full set of elements $D_{i j}(s)$ :

$$
D_{i j}(s)=\frac{(-1)^{i+j} \Pi_{j i}^{(N-1)}(s)}{\Pi^{(N)}(s)} .
$$

Here $\Pi^{(N)}(s)$ is the determinant of the matrix $\hat{D}^{-1}$, and $\Pi_{j i}^{(N-1)}(s)$ is a matrix supplement to the element $\left[\hat{D}^{-1}\right]_{j i}$, i.e. the matrix $\hat{D}^{-1}$ with the excluded $j$-th line and $i$-th column.

The zeros of $\Pi^{(N)}(s)$ define the poles of the propagator matrix which correspond to physical resonances formed by the mixing. We denote the complex resonance masses as:

$$
s=M_{A}^{2}, \quad M_{B}^{2}, \quad M_{C}^{2}, \ldots
$$

Near the point $s=M_{A}^{2}$, one can leave in the propagator matrix the leading pole term only. This means that the free term in Eq. (4.21) can be neglected, so we get a system of homogeneous equations:

$$
D_{i k}(s)\left(\hat{d}^{-1}-\hat{B}\right)_{k j}=0 .
$$

The solution of this system is defined up to the normalization factor, and it does not depend on the initial index $i$. Then the elements of the propagator matrix may be written in a factorized form as follows:

$$
\left[\hat{D}^{(N)}\right]_{s \sim M_{A}^{2}}=\frac{N_{A}}{M_{A}^{2}-s} \cdot\left|\begin{array}{llll}
\alpha_{1}^{2}, & \alpha_{1} \alpha_{2}, & \alpha_{1} \alpha_{3}, & \ldots \\
\alpha_{2} \alpha_{1}, & \alpha_{2}^{2}, & \alpha_{2} \alpha_{3}, & \ldots \\
\alpha_{3} \alpha_{1}, & \alpha_{3} \alpha_{2}, & \alpha_{3}^{2}, & \ldots \\
\ldots & \ldots & \ldots & \ldots
\end{array}\right|,
$$


where $N_{A}$ is the normalization factor chosen to satisfy the condition:

$$
\alpha_{1}^{2}+\alpha_{2}^{2}+\alpha_{3}^{2}+\ldots+\alpha_{N}^{2}=1 .
$$

The constants $\alpha_{i}$ are the normalized amplitudes for the transitions: resonance $A \rightarrow$ state $i$. The probability to find the state $i$ in the physical resonance $A$ is equal to:

$$
w_{i}=\left|\alpha_{i}\right|^{2} .
$$

Analogous representation of the propagator matrix can be also done in the vicinity of other poles:

$$
D_{i j}^{(N)}\left(s \sim M_{B}^{2}\right)=N_{B} \frac{\beta_{i} \beta_{j}}{M_{B}^{2}-s}, \quad D_{i j}^{(N)}\left(s \sim M_{C}^{2}\right)=N_{C} \frac{\gamma_{i} \gamma_{j}}{M_{C}^{2}-s} \quad \cdots
$$

Coupling constants satisfy normalization conditions similar to that of Eq. (4.28):

$$
\beta_{1}^{2}+\beta_{2}^{2}+\ldots+\beta_{N}^{2}=1, \quad \gamma_{1}^{2}+\gamma_{2}^{2}+\ldots+\gamma_{N}^{2}=1, \quad \cdots .
$$

In general case, however, there is no completness condition for the inverse expansion:

$$
\alpha_{i}^{2}+\beta_{i}^{2}+\gamma_{i}^{2}+\ldots \neq 1 \text {. }
$$

For two resonances, it means that $\cos ^{2} \Theta_{A}+\sin ^{2} \Theta_{B} \neq 1$. Still, let us remind that the equality in the inverse expansion, which is relevant to the completness condition, appears in the models, where the $s$-dependence of loop diagrams is neglected, see Eqs. (4.5)-(4.7).

\subsection{Full resonance overlapping: the accumulation of widths of neighbour- ing resonances by one of them}

Let us consider two examples which describe the idealized situation of a full overlapping of two or three resonances. In these examples, the effect of accumulation of widths of neighbouring resonances by one of them can be seen in its original untouched form.

a) Full overlapping of two resonances.

For the simplicity sake, let $B_{i j}$ be a weak $s$-dependent function, and Eq. (4.5) may be used. We define:

$$
M_{1}^{2}=M_{R}^{2}-i M_{R} \Gamma_{1}, \quad M_{2}^{2}=M_{R}^{2}-i M_{R} \Gamma_{2},
$$

and

$$
\operatorname{Re} B_{12}\left(M_{R}^{2}\right)=P \int_{\left(\mu_{1}+\mu_{2}\right)^{2}}^{\infty} \frac{d s^{\prime}}{\pi} \frac{g_{1}\left(s^{\prime}\right) g_{2}\left(s^{\prime}\right) \rho\left(s^{\prime}\right)}{s^{\prime}-M_{R}^{2}} \rightarrow 0 .
$$

It is possible that $\operatorname{Re} B_{12}\left(M_{R}^{2}\right)$ can be equal to zero at positive $g_{1}$ and $g_{2}$, if the contribution from the integration region $s^{\prime}<M_{R}^{2}$ cancels the contribution from the region $s^{\prime}>M_{R}^{2}$. In this case

$$
B_{12}\left(M_{R}^{2}\right) \rightarrow i g_{1}\left(M_{R}^{2}\right) g_{2}\left(M_{R}^{2}\right) \rho\left(M_{R}^{2}\right)=i M_{R} \sqrt{\Gamma_{1} \Gamma_{2}} .
$$

Substituting Eqs. (4.33)-(4.35) into Eq. (4.5), one has:

$$
M_{A}^{2} \rightarrow M_{R}^{2}-i M_{R}\left(\Gamma_{1}+\Gamma_{2}\right) \quad M_{B}^{2} \rightarrow M_{R}^{2} .
$$

Therefore, after mixing, one of the states accumulates the widths of primary resonances, $\Gamma_{A} \rightarrow \Gamma_{1}+\Gamma_{2}$, and another state becomes quasi-stable particle, with $\Gamma_{B} \rightarrow 0$.

b) Full overlapping of three resonances. 
Consider the equation

$$
\Pi^{(3)}(s)=0
$$

at the same approximation as in the above example. Correspondingly, we put:

$$
R e B_{a b}\left(M_{R}^{2}\right) \rightarrow 0,(a \neq b) ; \quad M_{i}^{2}=M_{R}^{2}-s-i M_{R} \Gamma_{i}=x-i \gamma_{i} .
$$

A new variable, $x=M_{R}^{2}-s$, is used, and we denote $M_{R} \Gamma_{i}=\gamma_{i}$. Taking account of $B_{i j} B_{j i}=-\gamma_{i} \gamma_{j}$ and $B_{12} B_{23} B_{31}=-i \gamma_{1} \gamma_{2} \gamma_{3}$, we can re-write the equation (4.37) as follows:

$$
x^{3}+x^{2}\left(i \gamma_{1}+i \gamma_{2}+i \gamma_{3}\right)=0
$$

Therefore, at full resonance overlapping, one obtains:

$$
M_{A}^{2} \rightarrow M_{R}^{2}-i M_{R}\left(\Gamma_{1}+\Gamma_{2}+\Gamma_{3}\right), \quad M_{B}^{2} \rightarrow M_{R}^{2}, \quad M_{C}^{2} \rightarrow M_{R}^{2}
$$

The resonance $A$ has accumulated the widths of three primary resonances, and the states $B$ and $C$ became quasi-stable and degenerate.

\subsection{The resonances $f_{0}(1300), f_{0}(1500), f_{0}\left(1530_{-250}^{+90}\right)$ and $f_{0}(1780)$}

The $K$-matrix analysis provides a basis for the investigation of mixing phenomenon in the scalar sector. Propagator matrix technique used at the next stage of the analysis allows us to restore correctly the contribution from the real parts of loop diagrams, $B_{i j}(s)$, thus having correctly calculated contributions of input states to the formation of physical resonances.

The resonance mixing in the region 1200-1600 MeV may be considered in the two-channel approximation, for the quark-hadron duality justifies the analysis with the use of quark channels $n \bar{n}$ and $s \bar{s}$. Correspondingly,

$$
B_{i j}(s)=\cos \varphi_{i} \cos \varphi_{j} B_{i j}^{(n \bar{n})}(s)+\sin \varphi_{i} \sin \varphi_{j} B_{i j}^{(s \bar{s})}(s),
$$

where $i, j$ run over $1,2,3,4$, with the following notations for states: $1=1^{3} P_{0}(n \bar{n}$ rich $), 2=$ $2^{3} P_{0}(n \bar{n}$ rich $), 3=$ gluonium and $4=2^{3} P_{0}(s \bar{s}$ rich $)$. The quark states are usually described by the light cone variables. Then,

$$
B_{i j}^{(n \bar{n})}(s)=\frac{1}{(2 \pi)^{3}} \int_{0}^{1} \frac{d x}{x} \int d^{2} k_{\perp} \frac{g_{i}\left(s^{\prime}\right) g_{j}\left(s^{\prime}\right)}{s^{\prime}-s} 2\left(s^{\prime}-4 m^{2}\right) .
$$

Here $s^{\prime}=\left(m^{2}+k_{\perp}^{2}\right) / x(1-x)$ and $m$ is the mass of nonstrange quark. The factor $2\left(s^{\prime}-4 m^{2}\right)$ appears due to the presence of the quark spin variables: $\operatorname{Tr}[(\hat{k}+m)(-\hat{p}+\hat{k}+m)]=2\left(s^{\prime}-4 m^{2}\right)$. Analogous expression, with the replacement $m \rightarrow m_{s}$, determines $B_{i j}^{(s \bar{s})}(s)$.

The simplest parametrization of vertex function for the transition input state $i \rightarrow$ quarks is:

$$
g_{i}(s)=\gamma_{i} \sqrt[4]{s}\left[\frac{k_{i}^{2}+\sigma_{i}}{k^{2}+\sigma_{i}}-d_{i} \frac{k_{i}^{2}+\sigma_{i}}{k^{2}+\sigma_{i}+h}\right] ;
$$

Here $k^{2}=s / 4-m^{2}$ and $k_{a}^{2}=m_{a}^{2} / 4-m^{2}$, where $m$ is the constituent quark mass equal to $350 \mathrm{MeV}$ for nonstrange quark and $500 \mathrm{MeV}$ for strange one, and $m_{a}$ is the mass of input state.

For the first state, $1^{3} P_{0}(n \bar{n}$ rich $)$, and for the gluonium we put $d_{1}=d_{3}=0$. The second state is the radial excitation, $2^{3} P_{0}(n \bar{n}$ rich $)$, and its wave function is orthogonal to the ground state. This means that real part of the function $B_{12}(s)$ must tend to zero at $s$ close to resonance masses. Such an orthogonalization has been performed at the point $\sqrt{s}=1.5 \mathrm{GeV}$, thus determining the value of the coefficient $d_{2}$. Vertex functions for the same nonet members are equal to each other, so $g_{2}(s)=g_{4}(s)$. 
Parameters $m_{a}, \gamma_{a}, h$ and $\sigma_{a}$ are defined by the masses and widths of physical resonances. However, the mass $m_{a}$ can be approximately fixed by the $K$-matrix pole: $\mu_{a}^{2} \simeq m_{a}^{2}-\operatorname{Re} B_{a a}\left(\mu_{a}^{2}\right)$. It should be underlined that $m_{3}$ is the mass of a pure gluonium which is a subject of the Lattice QCD.

Positions of amplitude poles and the masses of input states found in Ref. [7] by fitting the $00^{++}$ wave are shown in Table 4 . Relative weight of the primary state in the physical resonance $A$ is defined by Eq. (4.29): calculated in such a way the probabilities $W_{a}$ for the resonances under investigation are shown in Table 4.

As was stressed above, in order to make comparisons with the QCD calculations, one should separate the contributions from large and small distances, that is, to take into account the short-range interaction component, $r<r_{0} \sim R_{\text {confinement }}$, and eliminate the contribution from large $r$. Therefore, in the calculation of masses which might be compared with the results of the QCD-motivated models, we should make a replacement in the amplitude of the $00^{++}$wave as follows:

$$
B_{a b}(s) \rightarrow \operatorname{Re} \bar{B}_{a b}\left(s, k_{0}^{2}\right)=P \int_{4 m^{2}+4 k_{0}^{2}}^{\infty} \frac{d s^{\prime}}{\pi} \frac{g_{a}\left(s^{\prime}\right) \rho\left(s^{\prime}\right) g_{b}\left(s^{\prime}\right)}{s^{\prime}-s} 2\left(s^{\prime}-4 m^{2}\right) .
$$

The poles of the amplitude re-determined in this way provide the masses which are related to the interaction at $r<1 / k_{0}$. One must compare with the quark model results the masses obtained with a cutting, of the order of $k_{0}^{2} \sim 0.125(\mathrm{GeV} / \mathrm{c})^{2}$, that corersponds to the account of the quark interaction at $r \leq 1 \mathrm{fm} \sim R_{\text {confinement. }}$.

For Solution I we get (the values are given in $\mathrm{GeV}$ ):

$\begin{array}{lcccc} & 1^{3} P_{0}(s \bar{s} \text { rich }) & 1^{3} P_{0}(n \bar{n} \text { rich }) & 2^{3} P_{0}(n \bar{n} \text { rich }) & 2^{3} P_{0}(s \bar{s} \text { rich }) \\ m\left(k_{0}^{2}=0\right)=\mu_{a}^{\text {bare }} & 0.720 & 1.360 & 1.577 & 1.791 \\ m\left(k_{0}^{2}=0.125\right) & 0.730 & 1.340 & 1.560 & 1.780 \\ m\left(k_{0}^{2} \rightarrow \infty\right)=m_{a} & - & 1.457 & 1.536 & 1.750\end{array}$

In Solution II:

$\begin{array}{lcccc} & 1^{3} P_{0}(s \bar{s} \text { rich }) & 1^{3} P_{0}(n \bar{n} \text { rich }) & 2^{3} P_{0}(n \bar{n} \text { rich }) & 2^{3} P_{0}(s \bar{s} \text { rich }) \\ m\left(k_{0}^{2}=0\right)=\mu_{a}^{\text {bare }} & 0.720 & 1.357 & 1.585 & 1.734 \\ m\left(k_{0}^{2}=0.125\right) & 0.735 & 1.340 & 1.570 & 1.725 \\ m\left(k_{0}^{2} \rightarrow \infty\right)=m_{a} & - & 1.107 & 1.566 & 1.702\end{array}$

The lightest $q \bar{q}$-state, $f_{0}^{\text {bare }}(720)$, has not been included into the mixing machinary in Refs. [6, 7]. In Eqs. (4.45) and (4.46) the mass corrections for this state have been evaluated as $m_{a}\left(k_{0}^{2}\right) \simeq$ $m_{a}^{2}-\operatorname{Re} \bar{B}_{a a}\left(m_{a}^{2}, k_{0}^{2}\right)$. This approximate equality is due to a comparative smallness of the nondiagonal loop diagrams.

Equations (4.45) and (4.46) prove that the values $m_{a}\left(k_{0}^{2}=0.125 \mathrm{GeV}^{2}\right)$ slightly differ from $\mu_{a}^{\text {bare }}$, while the differences from the input masses $m_{a}$ can be significant. This means that the $K$-matrix analysis provides approximately correct meson characteristics which may be compared with the quark model results. On the contrary, one should compare with the results of Lattice QCD the values of input masses $m_{a}$, which can noticeably differ both from the masses of bare states, $\mu_{a}^{b a r e}$, and from those of real resonances.

\subsection{Dynamics of glueball mixing with $q \bar{q}$-states}

To find out the glueball mixing with $q \bar{q}$ - states, let us make a replacement in loop diagrams of the propagator matrix:

$$
g_{a}(s) \rightarrow \xi g_{a}(s),
$$


with the factor $\xi$ changing in the interval $0 \leq \xi \leq 1$. At $\xi \rightarrow 0$ the mixing is switched off, and the amplitude has the poles at $s \simeq m_{a}^{2}$. Figure 15 demonstrates the position of poles at different $\xi$ for Solutions I and II. With increasing $\xi$, the poles move from the real axis to the lower part of the complex plane. Let us discuss in detail the dynamics of the pole movement for Solution II.

At $\xi=0.1-0.5$ the glueball state of Solution II is mainly mixed with the state $2^{3} P_{0}(n \bar{n}$ rich $)$, while at $\xi=0.8-1.0$ the mixing with the state $1^{3} P_{0}(n \bar{n}$ rich becomes important. As a result, the state, which is the glueball descendant, sunk rather deeply into complex plane, acquiring the mass $M=1450-i 450 \mathrm{MeV}$, and the glueball component of this broad resonance is $47 \%$. Likewise, in Solution I the broad resonance is the gluon descendant as well.

The hypothesis about strong mixing of the gluonium with the $q \bar{q}$-states was raised formerly. But the attempts to restore quantitatively the picture of mixing within the standard quantum mechanics approach failed, for two phenomena have been lost:

(1) The $q \bar{q} /$ glueball mixing described by the $D$-matrix may lead not only to the repulsion of levels, that follows from the standard quantum mechanical approach, but to the attraction of levels as well. The latest effect is caused by the presence of imaginary parts of the loop diagrams $B_{a b}$, and it is important that $\operatorname{Im} B_{a b}$ is not small near $1500 \mathrm{MeV}$.

(2) The resonance overlapping leads to the repulsion of poles located on the imaginary axis of masses, and one resonance accumulates the widths of the others.

This very type of the mixing occurred at $1500 \mathrm{MeV}$, and a large width of one of the resonances is its inevitable consequence.

It is also natural that the broad resonance itself is the gluonium descendant, for the gluonuim mixes without any significant suppression with the nearby $q \bar{q}$-states, both of them being dominantly nonstrange ones. 
Table 4

Masses and mixing angles of the input states, the content of physical states and positions of poles of the $00^{++}$-amplitude (masses in $\mathrm{GeV}$ ).

\begin{tabular}{|c|c|c|c|c|}
\hline \multicolumn{5}{|c|}{ Solution I } \\
\hline & $\begin{array}{c}1^{3} P_{0}(n \bar{n} \text { rich }) \\
\phi_{1}=18^{\circ} \\
m_{1}=1.457\end{array}$ & $\begin{array}{c}2^{3} P_{0}(n \bar{n} \text { rich }) \\
\phi_{2}=-6^{\circ} \\
m_{2}=1.536\end{array}$ & $\begin{array}{c}\text { Gluonium } \\
\phi_{3}=25^{\circ} \\
m_{3}=1.230\end{array}$ & $\begin{array}{c}2^{3} P_{0}(s \bar{s} \text { rich }) \\
\phi_{4}=84^{\circ} \\
m_{4}=1.750 \\
\end{array}$ \\
\hline$W\left[f_{0}(1300)\right]$ & $32 \%$ & $12 \%$ & $55 \%$ & $1 \%$ \\
\hline $\begin{array}{c}1.300-i 0.115 \\
W\left[f_{0}(1500)\right] \\
1.500-i 0.065\end{array}$ & $25 \%$ & $70 \%$ & $3 \%$ & $2 \%$ \\
\hline$W\left[f_{0}(1530)\right]$ & $44 \%$ & $24 \%$ & $27 \%$ & $4 \%$ \\
\hline $\begin{array}{c}1.450-i 0.450 \\
W\left[f_{0}(1780)\right] \\
1.780-i 0.085\end{array}$ & $1 \%$ & $1 \%$ & $0 \%$ & $98 \%$ \\
\hline \multicolumn{5}{|c|}{ Solution II } \\
\hline & $\begin{array}{c}1^{3} P_{0}(n \bar{n} \text { rich }) \\
\phi_{1}=18^{\circ} \\
m_{1}=1.107\end{array}$ & $\begin{array}{c}2^{3} P_{0}(n \bar{n} \text { rich }) \\
\phi_{2}=35^{\circ} \\
m_{2}=1.566\end{array}$ & $\begin{array}{c}\text { Gluonium } \\
\phi_{3}=25^{\circ} \\
m_{3}=1.633\end{array}$ & $\begin{array}{c}2^{3} P_{0}(s \bar{s} \text { rich }) \\
\phi_{4}=-55^{\circ} \\
m_{4}=1.702\end{array}$ \\
\hline $\begin{array}{c}W\left[f_{0}(1300)\right] \\
1.300-i 0.115\end{array}$ & $35 \%$ & $26 \%$ & $38 \%$ & $0.4 \%$ \\
\hline $\begin{array}{c}W\left[f_{0}(1500)\right] \\
1.500-i 0.065\end{array}$ & $1 \%$ & $64 \%$ & $35 \%$ & $0.4 \%$ \\
\hline $\begin{array}{c}W\left[f_{0}(1530)\right] \\
1.450-i 0.450\end{array}$ & $12 \%$ & $41 \%$ & $47 \%$ & $0.3 \%$ \\
\hline $\begin{array}{c}W\left[f_{0}(1780)\right] \\
1.750-i 0.100\end{array}$ & $0.1 \%$ & $0.2 \%$ & $0.2 \%$ & $99.5 \%$ \\
\hline
\end{tabular}




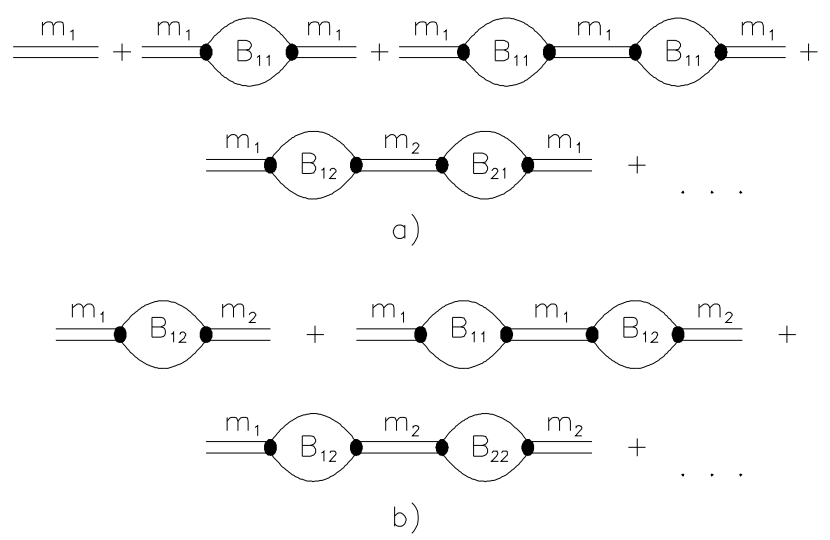

Fig. 14. Diagrams describing the propagation functions $D_{11}$ (a) and $D_{12}$ (b) for the interaction of two bare states. 

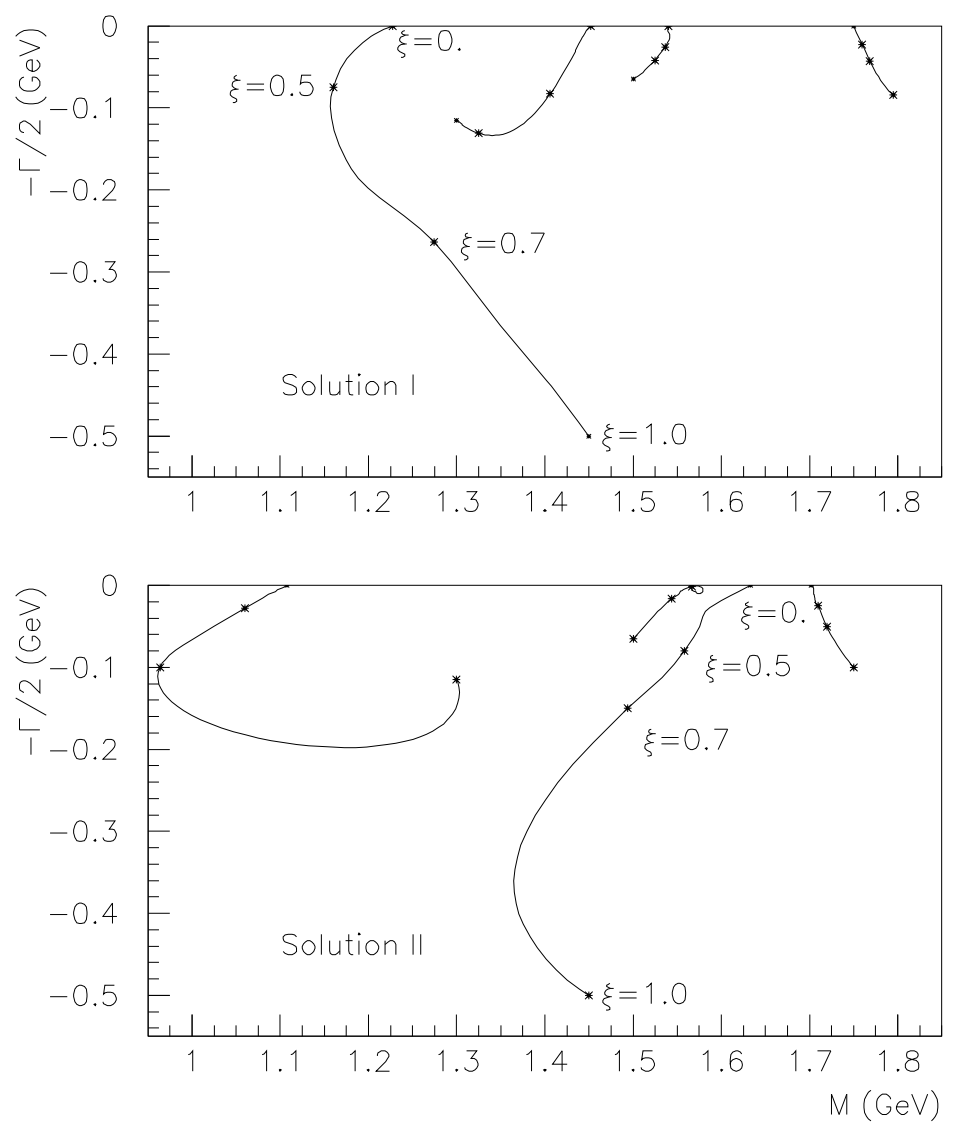

Fig. 15. Variation of the resonance pole positions plotted on the complex- $M$ plane at different coupling constants $g_{a} \rightarrow \xi g_{a}$.

\section{Conclusion}

Deconfinement of quarks from the excited $q \bar{q}$-levels is going in two stages:

1) Unavoidable production of $q \bar{q}$-pairs, which form two or more white states (hadrons).

2) The flying away of the produced hadrons, their interaction and, as a result, the mixing of neighbouring $q \bar{q}$-levels that leads to the formation of a broad locking state, which plays the role of a dynamical barrier for neighbouring levels.

That is the $K$-matrix analysis together with the dispersion $N / D$ method, which are summoned to decypher the second stage of the deconfinement. Analysis of $00^{++}$-wave carried out in the $K$-matrix or propagator matrix techniques demonstrated that the lightest scalar glueball, being near the states $1^{3} P_{0} q \bar{q}$ and $2^{3} P_{0} q \bar{q}$, turned after the mixing into a broad state, with $\Gamma / 2 \simeq 500 \mathrm{MeV}$. This broad state $f_{0}\left(1530_{-250}^{+90}\right)$ carries about a half of the scalar gluonium component, while the other components of the broad resonance are $1^{3} P_{0} q \bar{q}$ and $2^{3} P_{0} q \bar{q}$.

It looks like the waves $00^{-+}$and $02^{++}$behave similarly [57], that allows us to believe that physics 
of highly excited states is tightly connected with the study of broad states, being an important and inevitable step in the search for exotic hadrons and the investigation of confinement phenomenon.

Thanks are due to L.G. Dakhno and V.A. Nikonov for help. This investigation is supported by RFBR grant N 96-02-17934 and the INTAS-RFBR grant N 95-0267.

\section{References}

[1] Fritzsch H, Gell-Mann M in: Proc. of the XVI Int. Conf. on High Energy Physics, Batavia vol. 2, 135 (1972); Fritzsch H, Minkowski P Nuovo Cim. A30, 393 (1975); Freud P G O, Numbu Y Phys. Rev. Lett. 34, 1645 (1975).

[2] Jaffe R L, Johnson K Phys. Lett. B 60, 201 (1976).

[3] Bali G S, et al. Phys. Lett. B309 378 (1993).

[4] Sexton J, Vaccarino A, Weingarten D Phys. Rev. Lett. 75, 4563 (1995).

[5] Morningstar C J, Peardon M "Efficient glueball simulations on anisotropic lattices", heplat/9704011 (1997).

[6] Anisovich A V, Anisovich V V, Prokoshkin Yu D, Sarantsev A V, Z. Phys. A357, 123 (1997).

[7] Anisovich A V, Anisovich V V, Sarantsev A V, Phys. Lett. B395 123 (1997); Z. Phys. A359 173 (1997).

[8] t'Hooft G Nucl. Phys. B72 461 (1974); Veneziano G Nucl. Phys. B117 519 (1976).

[9] Particle Data Group: Barnet R M, et al. Phys. Rev. D54, 1 (1996).

[10] Heusch C A " Gluonium - an unfulfilled promise of QCD?" in: QCD-20 Years Later (Eds. Zerwas P M, Kastrup H A) (Singapore: World Scientific, 1993).

[11] Savinov V (CLEO Collaboration). "A measurement of the form factors of light pseudoscalar mesons at large momentum transfered" hep-ex/9507005 (1995); Behrend H J, et al. (CELLO Collaboration) Z. Phys. C49, 401 (1991); Aihara H, et al. (TCP $/ 2 \gamma$ Collaboration). Phys. Rev. Lett. 64, 172 (1990).

[12] Anisovich V V, Melikhov D I, Nikonov V A Phys. Rev. D55, 2918 (1997); Anisovich V V, Bugg D V, Melikhov D I, Nikonov V A Phys. Lett. B404, 166 (1997).

[13] Anisovich V V, Physics-Uspekhi 38, 1179 (1995).

[14] Anisovich V V, Kondashov A A, Prokoshkin Yu D, Sadovsky S A, Sarantsev A V Phys. Lett. B355, 363 (1995).

[15] Aker E, Amsler C, Augustin I, et al. (Crystal Barrel Collaboration) Phys. Lett. B260, 249 (1991).

[16] Anisovich V V, Bugg D V, Sarantsev A V, Zou B S Proceedings of Second Biennial Workshop on Nucleon-Antinucleon Physics (NAN'93), Moscow, 1993; Phys. Atomic Nuclei57, 1595 (1994).

[17] Anisovich V V, Bugg D V, Sarantsev A V, Zou B S Phys. Rev.D50, 1972 (1994). 
[18] Anisovich V V, Armstrong D S, Augustin I, et al. (Crystal Barrel Collaboration) Phys. Lett. B323, 233 (1994).

[19] Amsler C, Anisovich V V, Augustin I, et al. (Crystal Barrel Collaboration) Phys. Lett. B333, 277 (1994).

[20] Amsler C, Close F E Phys. Rev. DD53, 295 (1996); Phys. Lett. B353, 385 (1995).

[21] Close F E, Farrar G, Li Z P "Determining the gluonic content of isoscalar mesons" hep$\mathrm{ph} / 9610280$ (1996).

[22] Weingarten D "Scalar quarkonium and scalar glueball" hep-lat/9608070 (1996).

[23] Genovese M "A unified picture of glueball candidates $f_{0}(1500)$ and $f_{0}(1700)$ " Phys. Rev. $D$ to be published.

[24] Alde D, et al. Z. Phys. C66, 375 (1995); Prokoshkin Yu D, et al. Physics-Doklady 342, 473 (1995).

[25] Binon F, et al. Nuovo Cim. A78, 313 (1983).

[26] Binon F, et al. Nuovo Cim. A80, 363 (1984).

[27] Hyams B, et al. Nucl. Phys. B64, 134 (1977).

[28] Lindenbaum S J, Longacre R S Phys. Lett. B274, 492 (1992); Etkin A, et al. Phys. Rev. D25, 1786 (1982).

[29] Anisovich V V, Sarantsev A V Phys. Lett. B 382, 429 (1996).

[30] Anisovich V V, Prokoshkin Yu D, Sarantsev A V Phys. Lett. 389, 388 (1996).

[31] Alston-Garnjost M, et al. Phys. Lett. B36, 152 (1971).

[32] V.V. Anisovich V V, Huber M G, Kobrinsky M N, Metsch B Ch Phys. Rev. D42, 3045 (1990).

[33] Klempt E, Peters K Phys. Lett. BB352, 467 (1995).

[34] Anisovich V V Phys. Lett. B364, 195 (1995).

[35] Zou B S, private communication (1996).

[36] Anisovich V V, Kondashov A A, Prokoshkin Yu D, Sadovsky S A, Sarantsev A V "The two-pion spectra for the reaction $\pi^{-} p \rightarrow \pi^{0} \pi^{0} n$ at $38 \mathrm{GeV} / c$ pion momentum and combined analysis of the GAMS, Crystal Barrel and BNL data", hep-ph/9711319.

[37] Aston D, et al. Nucl. Phys. B296, 493 (1988).

[38] Weinstein J, Isgur N Phys. Rev. Lett. 48, 659 (1982); Phys. Rev. D27, 588 (1983); 41, 2236 (1990); Lohse D, et al. Nucl. Phys. A516, 513 (1990); Janssen G, et al. Phys. Rev. D52, 2690 (1995).

[39] Jaffe R L Phys. Rev. D15, 267 (1977); Achasov N N, Shestakov G N Z. Phys. C41, 309 (1988).

[40] Ritter Ch, Metsch B Ch, Muenz C R, Petry H R Phys. Lett. B380, 431 (1996).

[41] Close F E, Dokshitzer Yu, Gribov V N, et al. Phys. Lett. B319, 291 (1993). 
[42] Anisovich A V, Sarantsev A V "K-matrix analysis of the $K \pi$ S-wave in the mass region 900-2000 MeV and nonet classification of the scalar qu states", hep-ph/9705401; Phys. Lett. B in press.

[43] Chew G F, Mandelstam S Phys. Rev. 119, 467 (1960).

[44] Anisovich V V, Kobrinsky M N, Melikhov D I, Sarantsev A V Nucl. Phys. A544, 747 (1992); Anisovich V V, Melikhov D I, Metsch B Ch, Petry H R Nucl. Phys. A563, 549 (1993).

[45] Anisovich A V, Sarantsev A V Sov. J. Nucl. Phys. 55, 1200 (1992); Anisovich A V, Sadovnikova V A Sov. J. Nucl. Phys. 55, 1483 (1992).

[46] Kapitanov A V, Sarantsev A V Sov. J. Nucl. Phys. 56, 156 (1993).

[47] Anisovich V V, Shekhter V M Nucl. Phys. B 55, 455 (1973); Bjorken J D, Farrar G E Phys. Rev. $D \mathbf{9}, 1449$ (1974).

[48] Voloshin M A, Nikitin Yu P, Porfirov P I Sov. J. Nucl. Phys. 36, 586 (1982).

[49] Gershtein S S, Likhoded A K, Prokoshkin Yu D Z. Phys. C24, 305 (1984).

[50] Anisovich A V "Three-body dispersion relation equations for the coupled decay channels pp $\rightarrow$ $\pi \pi \pi, \eta \pi \pi, K \bar{K} \pi$ " hep-ph/9610523 (1996); Anisovich A V Sov. J. Nucl. Phys 58, 1467 (1995); Anisovich A V, Leutwyler H Phys. Lett. B375, 335 (1996).

[51] Bugg D V, Zou B S Phys. Rev. D50, 591 (1994); Morgan D, Pennington M R Phys. Rev. D48, 1185 (1993).

[52] Flatté W Phys. Lett. B63, 224 (1976).

[53] Kondashov A A, et al. Proc. 27th Intern. Conf. on High Energy Physics, Glasgow (1994) p. 1407; Kondashov A A, et al. Preprint IHEP 95-137, Protvino (1995).

[54] Zheng Z P In: Lepton and Photon Interactions, (Eds. Drell P, Rubin D) AIP Conf. Proc. 302 p. 530, AIP, New-York, 1994.

[55] Amsler C, et al. Phys. Lett. B342, (1995) 433; 355425 (1995);

[56] Bugg D V, Anisovich V V, Sarantsev A V, Zou B S Phys. Rev. D50, 4412 (1994); Bugg D V, Sarantsev A V, Zou B S Nucl. Phys. 471, 59 (1996).

[57] Bugg D V, Private communication 